\title{
Informing the public health response to COVID-19: a systematic review of risk factors for disease, severity, and mortality
}

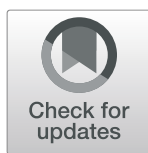

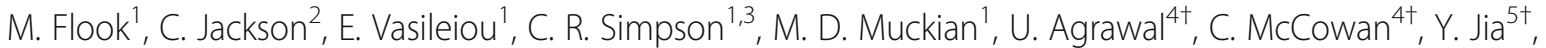 \\ J. L. K. Murray ${ }^{6,7 \dagger}$, L. D. Ritchie ${ }^{8 \dagger}$, C. Robertson ${ }^{9 \dagger}$, S. J. Stock ${ }^{1+}$, X. Wang ${ }^{1+}$, M. E. J. Woolhouse ${ }^{1,10 \dagger}$, A. Sheikh ${ }^{1}$ and \\ H. R. Stagg ${ }^{1 *}$ (D)
}

\begin{abstract}
Background: Severe Acute Respiratory Syndrome coronavirus-2 (SARS-CoV-2) has challenged public health agencies globally. In order to effectively target government responses, it is critical to identify the individuals most at risk of coronavirus disease-19 (COVID-19), developing severe clinical signs, and mortality. We undertook a systematic review of the literature to present the current status of scientific knowledge in these areas and describe the need for unified global approaches, moving forwards, as well as lessons learnt for future pandemics.

Methods: Medline, Embase and Global Health were searched to the end of April 2020, as well as the Web of Science. Search terms were specific to the SARS-CoV-2 virus and COVID-19. Comparative studies of risk factors from any setting, population group and in any language were included. Titles, abstracts and full texts were screened by two reviewers and extracted in duplicate into a standardised form. Data were extracted on risk factors for COVID-19 disease, severe disease, or death and were narratively and descriptively synthesised.

Results: One thousand two hundred and thirty-eight papers were identified post-deduplication. Thirty-three met our inclusion criteria, of which 26 were from China. Six assessed the risk of contracting the disease, 20 the risk of having severe disease and ten the risk of dying. Age, gender and co-morbidities were commonly assessed as risk factors. The weight of evidence showed increasing age to be associated with severe disease and mortality, and general comorbidities with mortality. Only seven studies presented multivariable analyses and power was generally limited. A wide range of definitions were used for disease severity.

(Continued on next page)
\end{abstract}

\footnotetext{
* Correspondence: helen.stagg@ed.ac.uk

†U. Agrawal, C. McCowan, Y. Jia, J. L. K. Murray, L. D. Ritchie, C. Robertson, S.

J. Stock, X. Wang and M. E. J. Woolhouse contributed equally to this work.

${ }^{1}$ Usher Institute, University of Edinburgh, 30 West Richmond Street,

Edinburgh EH8 9DX, UK

Full list of author information is available at the end of the article
}

(C) The Author(s). 2021 Open Access This article is licensed under a Creative Commons Attribution 4.0 International License, which permits use, sharing, adaptation, distribution and reproduction in any medium or format, as long as you give appropriate credit to the original author(s) and the source, provide a link to the Creative Commons licence, and indicate if changes were made. The images or other third party material in this article are included in the article's Creative Commons licence, unless indicated otherwise in a credit line to the material. If material is not included in the article's Creative Commons licence and your intended use is not permitted by statutory regulation or exceeds the permitted use, you will need to obtain permission directly from the copyright holder. To view a copy of this licence, visit http://creativecommons.org/licenses/by/4.0/ The Creative Commons Public Domain Dedication waiver (http://creativecommons.org/publicdomain/zero/1.0/) applies to the data made available in this article, unless otherwise stated in a credit line to the data. 


\begin{abstract}
(Continued from previous page)
Conclusions: The volume of literature generated in the short time since the appearance of SARS-CoV-2 has been considerable. Many studies have sought to document the risk factors for COVID-19 disease, disease severity and mortality; age was the only risk factor based on robust studies and with a consistent body of evidence. Mechanistic studies are required to understand why age is such an important risk factor. At the start of pandemics, large, standardised, studies that use multivariable analyses are urgently needed so that the populations most at risk can be rapidly protected.
\end{abstract}

Registration: This review was registered on PROSPERO as CRD42020177714.

Keywords: Coronavirus, COVID-19, Systematic review, Review, Risk factors, Morbidity, Mortality

\section{Introduction}

The world is currently experiencing a pandemic of coronavirus disease (COVID-19) caused by the Severe Acute Respiratory Syndrome coronavirus-2 (SARSCoV-2) [1]. The risk of morbidity and mortality from the virus is strongly stratified, with poor clinical outcomes considered more likely in certain vulnerable groups. For example, studies from different countries have established that older age groups are at increased risk of death [2, 3].

The ability to identity the population groups most at risk from the virus has manifold public health purposes. Using such data, stratified vaccination policies for governmental delivery can be designed, similar to those for influenza [4]. It may also be possible to prioritise more active monitoring of groups more at risk of clinical deterioration, and facilitate access to healthcare facilities by early identification of the individuals most likely to progress to severe disease who would thus be in need of intensive care and ventilation. Official advice can be issued to vulnerable groups to let them know that they are more at risk from SARS-CoV-2 virus, to promote behaviour modification $[5,6]$. Such population groups can also be the target of more formalised 'segment and shield' approaches: having divided the population into groups that present with similar health care concerns and needs (segmenting) it is possible to determine which groups require extra protection by reducing interaction with other groups (shielding), whilst relaxing restrictions for the rest of the population [7]. Potential public health policies along this route have been critiqued, however, on an inclusivity basis, particularly due to the unintended harmful consequences to already marginalised groups [8].

In the UK, vulnerable people were stratified into two tiers early on- 30th March 2020 (Table 1); those at risk of severe illness, who were advised to be particularly stringent with social distancing measures, and those within that group at further risk - described as 'shielded' individuals - who were advised to self-isolate and were provided with additional advice [9-12]. The former categorisation was based on the groups targeted for
National Health Service programmes on influenza vaccination and the latter on clinical consensus. These strata were deliberately broad, to maximise the number of individuals protected. As the evidence evolves - e.g. regarding whether the development of lesions in the cardiovascular system contributes meaningfully to disease pathogenesis in patients with and without pre-existing cardiovascular conditions [13] - there is the opportunity for the categorisation of risk of COVID-19 and serious outcomes from COVID-19 to become more evidencebased.

During epidemics and pandemics of emerging infectious diseases, it is critical to rapidly and accurately identify the populations most at risk. In the case of COVID19 , we undertook a systematic review and quality assessment of the rapidly-evolving global literature in this area, looking at three key outcomes: COVID-19 disease, disease severity, and mortality from the condition. Any potential risk factors, populations, and study designs were included. Arising from our findings, we highlight key knowledge gaps in the current literature and the need for unified global approaches moving forwards, particularly for the next pandemic.

\section{Materials and methods Literature search}

We systematically searched Medline, Embase, and Global Health (all via the Ovid platform), in addition to the Web of Science, for published literature between 1st November 2019 and 26th March 2020; then subsequently updated this search for a later period to 29th April. In order to avoid missing publications on risk factors, only terms specific to the virus and the disease were used, which were combined with 'or':

- 'coronavirus'

- 'covid-19'

- 'severe acute respiratory syndrome coronavirus 2'

- '2019-nCoV-2'

- 'SARS-CoV-2'

- 'acute respiratory syndrome' 
Table 1 UK risk groupings for COVID-19 disease on 30th March 2020

\begin{tabular}{ll}
\hline At risk of severe illness & Shielding \\
\hline $\begin{array}{l}\text { Aged } 70 \text { or older (regardless of medical conditions) } \\
\text { Aged under } 70 \text { and }\end{array}$ & \\
$\begin{array}{l}\text { Chronic (long-term) mild to moderate respiratory diseases, such as } \\
\text { asthma, COPD, emphysema or bronchitis }\end{array}$ & $\begin{array}{l}\text { People with severe chest conditions such as cystic fibrosis or severe } \\
\text { asthma (requiring hospital admissions or courses of steroid tablets) }\end{array}$ \\
$\begin{array}{l}\text { Chronic heart disease, such as heart failure } \\
\text { Chronic kidney disease }\end{array}$ & $\begin{array}{l}\text { People with severe diseases of body systems, such as severe kidney } \\
\text { disease (dialysis) }\end{array}$ \\
$\begin{array}{l}\text { Chronic liver disease, such as hepatitis } \\
\text { Chronic neurological conditions, such as Parkinson's disease, motor } \\
\text { neurone disease, MS, a learning disability or cerebral palsy }\end{array}$ & \\
$\begin{array}{l}\text { Diabetes } \\
\text { A weakened immune system as the result of conditions such as HIV } \\
\text { and AIDS, or medicines such as steroid tablets }\end{array}$ & $\begin{array}{l}\text { People who have received an organ transplant and remain on ongoing } \\
\text { immunosuppression medication }\end{array}$ \\
& $\begin{array}{l}\text { People with cancer who are undergoing active chemotherapy or } \\
\text { radiotherapy }\end{array}$ \\
& $\begin{array}{l}\text { People with cancers of the blood or bone marrow such as leukaemia who } \\
\text { are at any stage of treatment }\end{array}$
\end{tabular}

Being seriously overweight (a BMl of 40 or above)

Those who are pregnant

Data taken from sources [9-11]. ${ }^{\text {T}}$ These groupings represent individuals advised to get a yearly influenza vaccine as an adult for medical reasons. BMI body mass index, COPD chronic obstructive pulmonary disease, MS multiple sclerosis

No limits or filters were applied to the search. The same search terms were used across all databases.

Reference lists of included papers and review articles were also searched, as was the grey literature of public health reports for the 26 countries with the highest numbers of reported patients with COVID-19 at the end of April 2020, for other countries it was assumed there would be insufficient numbers of cases to yield relevant data.

\section{Eligibility criteria and study selection}

The following inclusion and exclusion criteria were applied to the search results.

Inclusion criteria:

- Studies had to provide comparative data on risk factors of any kind for disease (versus no disease), severe disease (versus milder disease) or mortality (versus survival),

- Studies were eligible if they presented data on patients with polymerase chain reaction (PCR)confirmed SARS-CoV-2 infections. There was considerable variation in case definitions between studies, but PCR testing was the gold standard test for active disease at the start of the pandemic [14], and other testing methods such as Loop-Mediated Isothermal Amplification or serological tests were not included,

- Any study design,
- Any population group,

- Any language of publication.

Exclusion criteria:

- No comparator group included in the study,

- Publication concerned other viruses and diseases,

- Work conducted in animals or in vitro,

- Study population was less than 20 individuals.

Two reviewers independently screened all titles, abstracts and full texts for both literature searches. Discrepancies were resolved by consensus. In all cases where studies were published in any language other than English, with no translations available, these were screened by at least one additional reviewer, with further quality control by another member of the reviewing team.

\section{Data extraction}

Three reviewers independently double-extracted the studies into a pre-designed spreadsheet that collected:

- First author,

- Paper title,

- Journal,

- Type of study,

- Country,

- Study population, 
- Overall number in study,

- Number with PCR confirmed SARS-CoV-2,

- Median age of participants/age range,

- Sex ratio,

- Analytical method used,

- Factors adjusted for during the analysis,

- Whether disease, disease severity, or death (or a combination of these) was the outcome of interest,

- The definition of disease severity used, if applicable,

- The risk factors analysed and the direction of effect.

Results were compared and discrepancies resolved by discussion. Data from studies published in languages other than English, at this stage only the Chinese language, were extracted by two additional reviewers, with further quality control by another member of the reviewing team.

\section{Quality assessment}

Two reviewers independently assessed the quality of included studies. Studies published in languages other than English were quality assessed by two additional reviewers, with further quality control by another member of the reviewing team. Assessments were undertaken from the perspective of the objectives of this review, which were not necessarily identical to the objectives of the underlying studies. The quality of included studies was assessed using a checklist adapted from Downs and Black [15], as per the guidance issued by Deeks et al. [16] When assessing the power of studies, the minimum sample size required to detect a relative increase in risk of $10 \%$ from a statistically conservative baseline of $50 \%$ among the unexposed was calculated at different powers using the Kelsey method within Epi Info, software made available by the United States Center for Disease Control [17]. This $10 \%$ value was based on governmental discussions taking place in the UK at the time the review took place. An alpha of 5\% was set as the standard. Pragmatically, we assumed only two strata and a ratio of 1:1 between exposure strata. Different thresholds were used for case-control studies and for cohort or cross-sectional studies. These criteria were scored from $0(<70 \%$ power $)$ to 5 (>99\% power). We considered results sufficient adjusted for confounding if they adjusted for at least the minimal variable set of age, sex, ethnicity and any measure of comorbidities. For ethnically homogenous populations, the need for adjustment for ethnicity was discounted. If two analyses were presented within a single paper with different quality scores, the most conservative score was retained. Studies were not excluded on the basis of the quality assessment.

\section{Analysis and synthesis}

Studies were grouped on the basis of the outcome examined (disease, disease severity, mortality) and then the risk factors examined. Results were classified on the basis of whether they presented evidence as to the exposure under study being a risk factor, taking into account the number of individuals exposed. Where studies focussed on a single risk factor of interest with adjustment for confounding, we extracted all data on potential risks in order to maximise the value of our dataset (whilst accepting that such mutually adjusted estimates for covariates may remain confounded even if that for the primary exposure does not) [18]. As there was substantial heterogeneity in study design, reporting, and the risk factors examined, we present a detailed descriptive summary and narrative synthesis of our findings, rather than a meta-analysis.

\section{Registration and reporting}

This review was registered on PROSPERO as CRD42020177714 and is reported according to the PRIS MA guidelines.

\section{Results}

Two thousand eight hundred and sixty-eight hits were obtained by the searches across the two dates (Fig. 1). After de-duplication across the different databases, this was reduced to 1238 . Thirty studies were included at the extraction stage; the main reasons for exclusion were small numbers of participants and studies not having a comparator population. From the grey literature an additional report was included and two studies were identified from reference lists.

Included studies are presented in Table 2. Twentynine of the 33 studies were conducted in China, with one each from France, Italy, Singapore and a combined study from England, Wales and Northern Ireland. Six were studies with COVID-19 disease as the outcome, 20 of disease severity and ten of mortality. One additional study looked at a combined outcome of disease severity and mortality.

\section{Quality assessment}

Included studies were generally too small to detect a $10 \%$ increase in risk of disease, disease severity, or mortality (Table 3). One study among the 33 was assessed to have 95\% power and two others $99 \%$; all were large, national, investigations. As 26 studies were purely descriptive or presented univariable analysis only, there was no adjustment for confounding. Remaining studies with a regression component did not adjust for our minimal confounder set. Only nine studies provided estimates of the random variability of effect estimates. The majority of studies ascertained exposure information from clinical records, which would have collected data prospectively and thus with limited recall bias. Blinding of outcome and exposure recording by investigators was not documented. In 


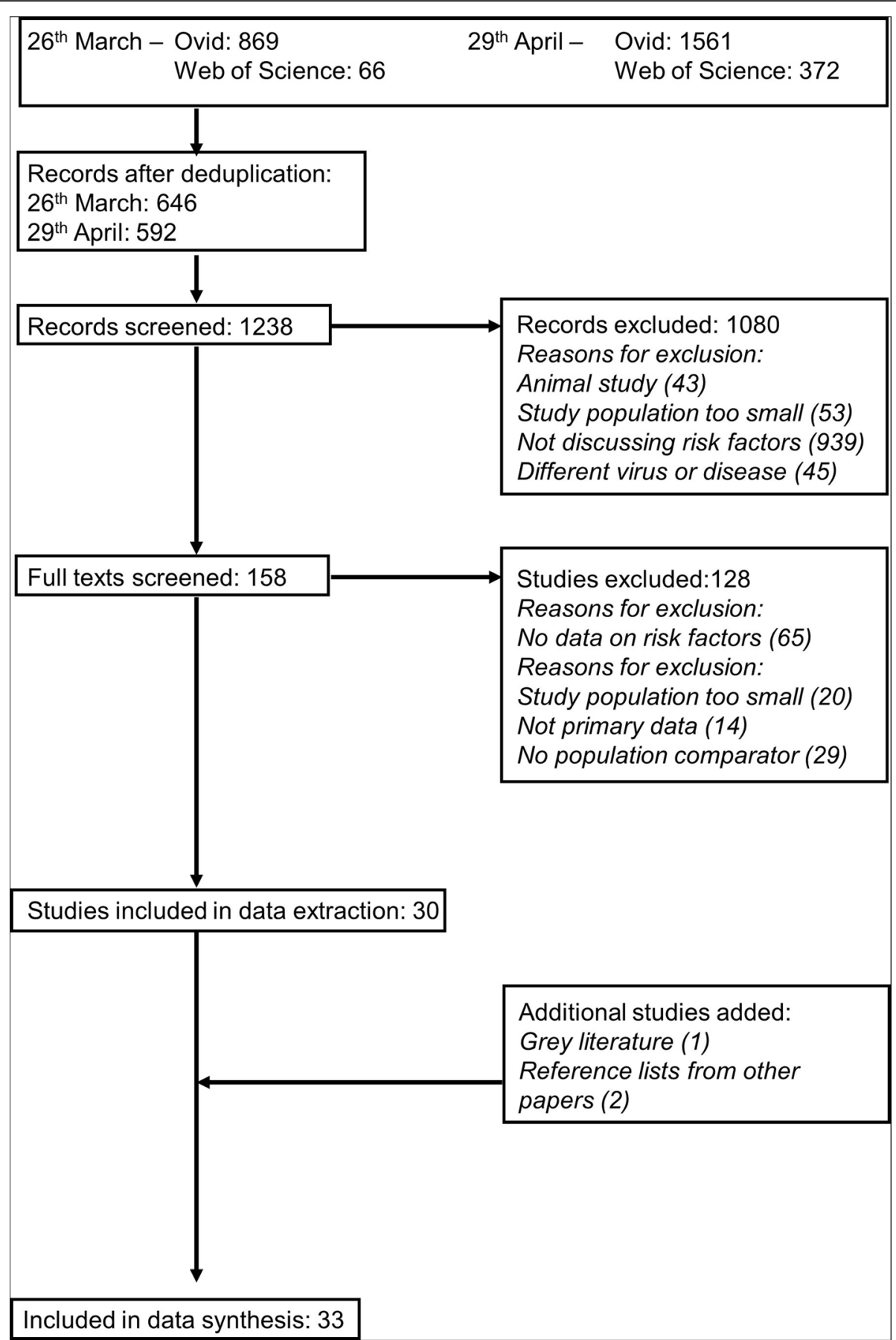

Fig. 1 PRISMA flow chart of selection

the case of certain disease severity outcomes, such as admittance to intensive care units (ICU), variability in thresholds for reaching these outcomes is likely to exist between settings and clinicians.

\section{Risk factors for disease}

Six studies compared the likelihood of having COVID-19 to other infectious conditions (Table 4).
Of note, as testing strategies were largely focussed on hospitalised individuals i.e. those displaying noticeable symptoms, studies were of the likelihood of COVID-19 disease, rather than more broadly of SARS-CoV-2 infection (and particularly of severe disease, although patients with mild and symptomatic infection were also reported to be hospitalised in some studies for the purposes of isolation or 
Table 2 Included studies

\begin{tabular}{|c|c|c|c|c|c|c|c|c|}
\hline Author & $\begin{array}{l}\text { Type of } \\
\text { study }\end{array}$ & Country & Study population & $\begin{array}{l}\text { Overall } \\
\text { number } \\
\text { of } \\
\text { patients }\end{array}$ & $\begin{array}{l}\text { Analytical } \\
\text { method } \\
\text { used }\end{array}$ & $\begin{array}{l}\text { Factors included } \\
\text { in multivariable } \\
\text { model }\end{array}$ & $\begin{array}{l}\text { Disease, } \\
\text { disease } \\
\text { severity, } \\
\text { or death }\end{array}$ & Definition of severity \\
\hline Cai [19] & $\begin{array}{l}\text { Retrospective } \\
\text { cohort }\end{array}$ & China & $\begin{array}{l}\text { Hospitalised patients, } \\
\text { single hospital }\end{array}$ & 298 & $\begin{array}{l}\text { Univariable; } \\
\text { logistic } \\
\text { regression }\end{array}$ & $\begin{array}{l}\text { N/A; age, sex, } \\
\text { comorbidities, } \\
\text { clinical markers, } \\
\text { travel history }\end{array}$ & $\begin{array}{l}\text { Disease } \\
\text { severity }\end{array}$ & $\begin{array}{l}\text { As per the international } \\
\text { guidelines for } \\
\text { community-acquired } \\
\text { pneumonia, with a } \\
\text { scoring system based } \\
\text { on demographics, co- } \\
\text { morbid illness, physical } \\
\text { examination findings, } \\
\text { and laboratory and } \\
\text { radiographic findings }\end{array}$ \\
\hline Chen [20] & $\begin{array}{l}\text { Retrospective } \\
\text { cohort }\end{array}$ & China & $\begin{array}{l}\text { Hospitalised patients, } \\
\text { single hospital }\end{array}$ & 249 & $\begin{array}{l}\text { Logistic } \\
\text { regression }\end{array}$ & $\begin{array}{l}\text { Age, sex, } \\
\text { comorbidities and } \\
\text { a range of clinical } \\
\text { measures }\end{array}$ & $\begin{array}{l}\text { Disease } \\
\text { severity }\end{array}$ & ICU admission \\
\hline Chen [21] & $\begin{array}{l}\text { Retrospective } \\
\text { cohort with a } \\
\text { nested case- } \\
\text { control study }\end{array}$ & China & $\begin{array}{l}\text { Hospitalised patients } \\
\text { (moderately, severely or } \\
\text { critically ill), single hospital }\end{array}$ & 274 & Univariable & N/A & Death & N/A \\
\hline Chen [22] & Case-control & China & $\begin{array}{l}\text { COVID-19 vs. pneumonia } \\
\text { negative for SARS-CoV-2 }\end{array}$ & $\begin{array}{l}104(78 \\
\text { COVID- } \\
19)\end{array}$ & Univariable & N/A & Disease & N/A \\
\hline Chen [23] & $\begin{array}{l}\text { Retrospective } \\
\text { cohort }\end{array}$ & China & $\begin{array}{l}\text { Hospitalised patients, } \\
\text { single hospital }\end{array}$ & 21 & Univariable & N/A & $\begin{array}{l}\text { Disease } \\
\text { severity }\end{array}$ & $\begin{array}{l}\text { Severe meets any of } \\
\text { the following criteria- } \\
\text { respiratory distress, } \\
\mathrm{RR} \geq 30 \text { breaths/min; } \\
\mathrm{SpO}_{2} \leq 93 \% \text { at rest; and } \\
\mathrm{PaO}_{2} / \mathrm{FIO}_{2} \leq 300 \text {. } \\
\text { Patients with greater } \\
\text { than } 50 \% \text { lesion } \\
\text { progression within } 24 \\
\text { to } 48 \mathrm{~h} \text { in pulmonary } \\
\text { imaging. }\end{array}$ \\
\hline Cheng [24] & Case-control & China & $\begin{array}{l}\text { Patients presenting with } \\
\text { fever diagnosed with } \\
\text { pneumonia by specialists } \\
\text { with chest CT scans }\end{array}$ & $\begin{array}{l}38(11 \\
\text { COVID- } \\
19)\end{array}$ & Univariable & N/A & Disease & N/A \\
\hline $\begin{array}{l}\text { Chinese } \\
\text { CDC [25] }\end{array}$ & $\begin{array}{l}\text { Retrospective } \\
\text { cohort }\end{array}$ & China & Nationwide surveillance & 72,314 & Univariable & N/A & Death & $\mathrm{N} / \mathrm{A}$ \\
\hline Fan [26] & $\begin{array}{l}\text { Retrospective } \\
\text { cohort }\end{array}$ & Singapore & $\begin{array}{l}\text { Hospitalised patients, } \\
\text { National Centre for } \\
\text { Infectious Diseases }\end{array}$ & 67 & Univariable & N/A & $\begin{array}{l}\text { Disease } \\
\text { severity }\end{array}$ & ICU admission \\
\hline $\begin{array}{l}\text { Grasselli } \\
{[27]}\end{array}$ & $\begin{array}{l}\text { Prospective } \\
\text { cohort with a } \\
\text { nested case- } \\
\text { control study }\end{array}$ & Italy & $\begin{array}{l}\text { Hospitalised in ICU across } \\
72 \text { hospitals }\end{array}$ & 1591 & Univariable & N/A & Death & N/A \\
\hline $\operatorname{Han}[28]$ & $\begin{array}{l}\text { Retrospective } \\
\text { cohort }\end{array}$ & China & $\begin{array}{l}\text { Hospitalised patients, } \\
\text { single hospital }\end{array}$ & 273 & Univariable & N/A & $\begin{array}{l}\text { Disease } \\
\text { severity }\end{array}$ & $\begin{array}{l}\text { Severe met at least one } \\
\text { of the following } \\
\text { conditions: a) shortness } \\
\text { of breath, RR } \geq 30 \\
\text { times/min, b) oxygen } \\
\text { saturation (resting } \\
\mathrm{state} \leq 93 \% \text {, or c) } \\
\mathrm{PaO}_{2} / \mathrm{FiO}_{2} \leq 300 \\
\mathrm{mmHg} \text {, in addition to } \\
\text { positive } \mathrm{SARS}-\mathrm{CoV}-2 \\
\mathrm{RNA} \text { nucleic acid test } \\
\text { by Reverse transcription } \\
\text { polymerase chain reac- } \\
\text { tion, fever, or other re- } \\
\text { spiratory symptoms }\end{array}$ \\
\hline
\end{tabular}


Table 2 Included studies (Continued)

\begin{tabular}{lllll}
\hline Author & $\begin{array}{l}\text { Type of } \\
\text { study }\end{array}$ & Country & Study population & $\begin{array}{l}\text { Overall } \\
\text { number } \\
\text { of } \\
\text { patients }\end{array}$ \\
& & $\begin{array}{l}\text { Analytical } \\
\text { method }\end{array}$ & $\begin{array}{l}\text { Factors included } \\
\text { in multivariable } \\
\text { model }\end{array}$ \\
\hline
\end{tabular}

\section{Disease, Definition of severity \\ disease \\ severity, \\ or death}

(the typical CT image abnormities of viral pneumonia were optional). Critical patients also needed to meet at least one of the extra following conditions: a) respiratory failure that needs to receive mechanical ventilation; b) shock; and c) multiple organ failure that need to be transferred to the ICU.

\begin{tabular}{|c|c|c|c|c|c|c|c|c|}
\hline $\begin{array}{l}\text { Huang } \\
{[29]}\end{array}$ & $\begin{array}{l}\text { Retrospective } \\
\text { cohort }\end{array}$ & China & $\begin{array}{l}\text { Hospitalised patients, } \\
\text { single hospital }\end{array}$ & 41 & Univariable & N/A & $\begin{array}{l}\text { Disease } \\
\text { severity }\end{array}$ & ICU admission \\
\hline $\begin{array}{l}\text { ICNARC } \\
{[30]}\end{array}$ & $\begin{array}{l}\text { Case-control } \\
\text { (disease)/ } \\
\text { Prospective } \\
\text { cohort } \\
\text { (disease } \\
\text { severity) }\end{array}$ & $\begin{array}{l}\text { England, } \\
\text { Northern } \\
\text { Ireland } \\
\text { and } \\
\text { Wales }\end{array}$ & $\begin{array}{l}\text { Hospitalised patients in } \\
\text { ICU across a network of } \\
\text { hospitals }\end{array}$ & $\begin{array}{l}12,502 \\
(6720 \\
\text { COVID- } \\
19)\end{array}$ & Univariable & N/A & $\begin{array}{l}\text { Disease; } \\
\text { Disease } \\
\text { severity }\end{array}$ & $\begin{array}{l}\text { Receiving advanced, as } \\
\text { opposed to basic, } \\
\text { respiratory support }\end{array}$ \\
\hline Liang [31] & $\begin{array}{l}\text { Prospective } \\
\text { cohort }\end{array}$ & China & $\begin{array}{l}\text { Hospitalised patients } \\
\text { across } 575 \text { hospitals }\end{array}$ & 1590 & $\begin{array}{l}\text { Logistic } \\
\text { regression; } \\
\text { Cox } \\
\text { regression }\end{array}$ & $\begin{array}{l}\text { Logistic model } \\
\text { adjusted for age, } \\
\text { sex, smoking } \\
\text { history, } \\
\text { comorbidities, } \\
\text { cancer }\end{array}$ & $\begin{array}{l}\text { Disease } \\
\text { severity, } \\
\text { Death }\end{array}$ & $\begin{array}{l}\text { Invasive ventilation, ICU } \\
\text { admission, death }\end{array}$ \\
\hline Liu [32] & $\begin{array}{l}\text { Retrospective } \\
\text { cohort }\end{array}$ & China & $\begin{array}{l}\text { Hospitalised patients, } \\
\text { single hospital }\end{array}$ & 119 & Univariable & N/A & $\begin{array}{l}\text { Disease } \\
\text { severity }\end{array}$ & $\begin{array}{l}\text { Severe- dyspnoea } \\
\text { accompanied by } \\
\text { hypoxemia, sometimes } \\
\text { acute respiratory } \\
\text { distress syndrome, } \\
\text { septic shock and } \\
\text { multiple organ failure }\end{array}$ \\
\hline Liu [33] & $\begin{array}{l}\text { Retrospective } \\
\text { cohort }\end{array}$ & China & $\begin{array}{l}\text { Hospitalised patients, } \\
\text { single hospital }\end{array}$ & 4880 & $\begin{array}{l}\text { Logistic } \\
\text { regression }\end{array}$ & Age, sex & Disease & N/A \\
\hline Qiu [34] & $\begin{array}{l}\text { Retrospective } \\
\text { cohort }\end{array}$ & China & $\begin{array}{l}\text { Hospitalised paediatric } \\
\text { patients, three hospitals }\end{array}$ & 36 & Univariable & N/A & $\begin{array}{l}\text { Disease } \\
\text { severity }\end{array}$ & $\begin{array}{l}\text { Moderate disease (mild } \\
\text { was baseline)- mild } \\
\text { pneumonia; symptoms } \\
\text { such as fever, cough, } \\
\text { fatigue, headache, and } \\
\text { myalgia; no } \\
\text { complications and } \\
\text { manifestations related } \\
\text { to severe conditions }\end{array}$ \\
\hline Ruan [35] & $\begin{array}{l}\text { Retrospective } \\
\text { cohort with a } \\
\text { nested case- } \\
\text { control study }\end{array}$ & China & $\begin{array}{l}\text { Hospitalised patients } \\
\text { across two hospitals with } \\
\text { a definitive outcome }\end{array}$ & 150 & Univariable & N/A & Death & N/A \\
\hline Shi [36] & $\begin{array}{l}\text { Retrospective } \\
\text { cohort }\end{array}$ & China & $\begin{array}{l}\text { Hospitalised patients } \\
\text { across a province }\end{array}$ & 487 & $\begin{array}{l}\text { Univariable; } \\
\text { logistic } \\
\text { regression }\end{array}$ & $\begin{array}{l}\text { N/A; age, sex, } \\
\text { hypertension; full } \\
\text { list unknown }\end{array}$ & $\begin{array}{l}\text { Disease } \\
\text { severity }\end{array}$ & $\begin{array}{l}\text { Severe pneumonia, } \\
\text { characterised by fever, } \\
\text { cough, dyspnoea, } \\
\text { bilateral pulmonary } \\
\text { infiltrates, and acute } \\
\text { respiratory injury }\end{array}$ \\
\hline $\begin{array}{l}\text { Simmonet } \\
\text { [37] }\end{array}$ & $\begin{array}{l}\text { Retrospective } \\
\text { cohort }\end{array}$ & France & $\begin{array}{l}\text { Hospitalised patients in } \\
\text { ICU, single hospital }\end{array}$ & 124 & $\begin{array}{l}\text { Logistic } \\
\text { regression }\end{array}$ & $\begin{array}{l}\text { Age, sex, diabetes, } \\
\text { hypertension, BMl }\end{array}$ & $\begin{array}{l}\text { Disease } \\
\text { severity }\end{array}$ & $\begin{array}{l}\text { Receiving invasive } \\
\text { mechanical ventilation, } \\
\text { determined when } \\
\text { oxygen therapy } \geq 10\end{array}$ \\
\hline
\end{tabular}


Table 2 Included studies (Continued)

\begin{tabular}{|c|c|c|c|c|c|c|c|c|}
\hline Author & $\begin{array}{l}\text { Type of } \\
\text { study }\end{array}$ & Country & Study population & $\begin{array}{l}\text { Overall } \\
\text { number } \\
\text { of } \\
\text { patients }\end{array}$ & $\begin{array}{l}\text { Analytical } \\
\text { method } \\
\text { used }\end{array}$ & $\begin{array}{l}\text { Factors included } \\
\text { in multivariable } \\
\text { model }\end{array}$ & $\begin{array}{l}\text { Disease, } \\
\text { disease } \\
\text { severity, } \\
\text { or death }\end{array}$ & Definition of severity \\
\hline & & & & & & & & $\begin{array}{l}\mathrm{L} / \mathrm{min} \text { ) with target } \\
\mathrm{SpO}_{2}(90-94 \%) \text { was } \\
\text { ineffective, and when } \\
\mathrm{RR} \text { was above } 25 / \mathrm{min} \text {, } \\
\text { with signs of acute } \\
\text { respiratory failure, } \\
\text { despite maximal } \\
\text { oxygen therapy }\end{array}$ \\
\hline Tian [38] & $\begin{array}{l}\text { Retrospective } \\
\text { cohort }\end{array}$ & China & $\begin{array}{l}\text { Hospitalised individuals } \\
\text { transferred from the } \\
\text { hospitals of Beijing to the } \\
\text { designated hospitals for } \\
\text { specialist treatment of } \\
\text { infectious diseases by } \\
\text { Beijing Emergency } \\
\text { Medical Service }\end{array}$ & 262 & Univariable & N/A & $\begin{array}{l}\text { Disease } \\
\text { severity }\end{array}$ & $\begin{array}{l}\text { Severe- dyspnoea or } \\
\text { respiratory failure in } \\
\text { addition to fever, } \\
\text { respiratory symptoms } \\
\text { and radiographic } \\
\text { evidence of pneumonia }\end{array}$ \\
\hline Wan [39] & $\begin{array}{l}\text { Prospective } \\
\text { cohort }\end{array}$ & China & $\begin{array}{l}\text { Hospitalised patients, } \\
\text { single hospital }\end{array}$ & 135 & Univariable & N/A & $\begin{array}{l}\text { Disease } \\
\text { severity }\end{array}$ & $\begin{array}{l}\text { Severe group- } \\
\text { respiratory distress, } \\
\text { RR } \geq 30 \text { breaths/minute } \\
\text { in a resting state, a } \\
\text { mean oxygen } \\
\text { saturation of } \leq 93 \% \text {, } \\
\text { and } a n \mathrm{PaO}_{2} / \mathrm{FiO}_{2} \leq \\
300 \mathrm{mmHg}\end{array}$ \\
\hline Wang [40] & Case-control & China & $\begin{array}{l}\text { Country-wide data } \\
\text { compared to historic } \\
\text { SARS/MERS }\end{array}$ & $\begin{array}{l}11,425 \\
(835 \\
\text { COVID- } \\
19)\end{array}$ & Univariable & N/A & Disease & N/A \\
\hline Wang [41] & $\begin{array}{l}\text { Retrospective } \\
\text { cohort }\end{array}$ & China & $\begin{array}{l}\text { Hospitalised patients, } \\
\text { single hospital }\end{array}$ & 138 & Univariable & N/A & $\begin{array}{l}\text { Disease } \\
\text { severity }\end{array}$ & ICU admission \\
\hline Wang [42] & $\begin{array}{l}\text { Prospective } \\
\text { cohort }\end{array}$ & China & $\begin{array}{l}\text { Hospitalised patients, } \\
\text { single hospital }\end{array}$ & 116 & Univariable & N/A & $\begin{array}{l}\text { Disease } \\
\text { severity }\end{array}$ & $\begin{array}{l}\text { Severe- fever or } \\
\text { suspected respiratory } \\
\text { infection, plus one of } \\
\text { the following: RR }>30 \\
\text { breaths/min; severe } \\
\text { respiratory distress; or } \\
\mathrm{SpO}_{2} \leq 93 \% \text { on room } \\
\text { air }\end{array}$ \\
\hline Wang [43] & $\begin{array}{l}\text { Cross- } \\
\text { sectional }\end{array}$ & China & $\begin{array}{l}\text { Hospitalised patients, } \\
\text { single hospital }\end{array}$ & 69 & Univariable & N/A & $\begin{array}{l}\text { Disease } \\
\text { severity }\end{array}$ & $\mathrm{SpO}_{2}<90 \%$ \\
\hline Wu [44] & $\begin{array}{l}\text { Retrospective } \\
\text { cohort }\end{array}$ & China & $\begin{array}{l}\text { Hospitalised patients, } \\
\text { single hospital }\end{array}$ & 201 & $\begin{array}{l}\text { Univariable; } \\
\text { Cox } \\
\text { regression }\end{array}$ & N/A & $\begin{array}{l}\text { Disease } \\
\text { severity, } \\
\text { Death }\end{array}$ & $\begin{array}{l}\text { ARDS, mechanical } \\
\text { ventilation }\end{array}$ \\
\hline Wu [45] & $\begin{array}{l}\text { Prospective } \\
\text { cohort }\end{array}$ & China & Country-wide data & 44,672 & Univariable & N/A & Death & N/A \\
\hline Yang [46] & $\begin{array}{l}\text { Retrospective } \\
\text { cohort }\end{array}$ & China & $\begin{array}{l}\text { Hospitalised patients, } \\
\text { single hospital, admitted } \\
\text { to ICU }\end{array}$ & 52 & $\begin{array}{l}\text { Univariable; } \\
\text { Cox } \\
\text { regression }\end{array}$ & N/A & Death & N/A \\
\hline Yuan [47] & $\begin{array}{l}\text { Retrospective } \\
\text { cohort }\end{array}$ & China & $\begin{array}{l}\text { Hospitalised patients, } \\
\text { single hospital }\end{array}$ & 27 & Univariable & N/A & Death & N/A \\
\hline Zhang [48] & $\begin{array}{l}\text { Retrospective } \\
\text { cohort }\end{array}$ & China & $\begin{array}{l}\text { Hospitalised patients, } \\
\text { single hospital }\end{array}$ & 140 & Univariable & N/A & $\begin{array}{l}\text { Disease } \\
\text { severity }\end{array}$ & $\begin{array}{l}\text { Severe COVID-19 was } \\
\text { designated when the } \\
\text { patients had one of the } \\
\text { following criteria: (a) re- } \\
\text { spiratory distress with } \\
\text { respiratory frequency } \geq \\
30 / \text { min; (b) pulse oxim- } \\
\text { eter oxygen satur- } \\
\text { ation } \leq 93 \% \text { at rest; and }\end{array}$ \\
\hline
\end{tabular}


Table 2 Included studies (Continued)

\begin{tabular}{|c|c|c|c|c|c|c|c|c|}
\hline Author & $\begin{array}{l}\text { Type of } \\
\text { study }\end{array}$ & Country & Study population & $\begin{array}{l}\text { Overall } \\
\text { number } \\
\text { of } \\
\text { patients }\end{array}$ & $\begin{array}{l}\text { Analytical } \\
\text { method } \\
\text { used }\end{array}$ & $\begin{array}{l}\text { Factors included } \\
\text { in multivariable } \\
\text { model }\end{array}$ & $\begin{array}{l}\text { Disease, } \\
\text { disease } \\
\text { severity, } \\
\text { or death }\end{array}$ & Definition of severity \\
\hline & & & & & & & & $\begin{array}{l}\text { (c) oxygenation index } \\
\left(\mathrm{PaO}_{2} / \mathrm{FiO}_{2}\right) \leq 300 \\
\mathrm{mmHg}\end{array}$ \\
\hline Zhang [49] & $\begin{array}{l}\text { Retrospective } \\
\text { cohort }\end{array}$ & China & $\begin{array}{l}\text { Hospitalised patients } \\
\text { (patients with no severe } \\
\text { underlying diseases), } \\
\text { single hospital }\end{array}$ & 95 & Univariable & N/A & $\begin{array}{l}\text { Disease } \\
\text { severity }\end{array}$ & $\begin{array}{l}\text { Severe- } \mathrm{RR} \geq 30 \text { times / } \\
\text { min; at rest, oxygen } \\
\text { saturation } \leq 93 \% ; \mathrm{PaO}_{2} / \\
\mathrm{FiO}_{2} \leq 300 \mathrm{mmHg}\end{array}$ \\
\hline Zhang [50] & $\begin{array}{l}\text { Retrospective } \\
\text { cohort }\end{array}$ & China & $\begin{array}{l}\text { Hospitalised patients, } \\
\text { single hospital }\end{array}$ & 120 & Univariable & N/A & $\begin{array}{l}\text { Disease } \\
\text { severity }\end{array}$ & $\begin{array}{l}\text { Severe- when patients } \\
\text { met one of the } \\
\text { following criteria: (1) } \\
\text { respiratory distress with } \\
\text { a breathing rate } \geq 30 / \\
\text { min; (2) pulse oximeter } \\
\text { oxygen saturation } \leq \\
93 \% \text { at rest; (3) } \\
\text { oxygenation index } \\
\left(\mathrm{PaO}_{2} / \mathrm{FiO}_{2}\right) \leq 300 \\
\mathrm{mmHg} \text { (4) respiratory } \\
\text { failure requiring } \\
\text { mechanical ventilation; } \\
\text { (5) shock; and (6) } \\
\text { combined with other } \\
\text { organ failure requiring } \\
\text { ICU monitoring and } \\
\text { treatment }\end{array}$ \\
\hline Zhou [51] & $\begin{array}{l}\text { Retrospective } \\
\text { cohort with a } \\
\text { nested case- } \\
\text { control study }\end{array}$ & China & $\begin{array}{l}\text { Hospitalised patients } \\
\text { across two hospitals with } \\
\text { a definitive outcome }\end{array}$ & 191 & $\begin{array}{l}\text { Univariable; } \\
\text { logistic } \\
\text { regression }\end{array}$ & $\begin{array}{l}\text { N/A; age, coronary } \\
\text { heart disease, } \\
\text { Sequential Organ } \\
\text { Failure Assessment } \\
\text { score, lymphocyte } \\
\text { count, D-dimer }\end{array}$ & Death & $\mathrm{N} / \mathrm{A}$ \\
\hline
\end{tabular}

ARDS acute respiratory distress syndrome, $B M I$ body mass index, $C D C$ Center for Disease Control and Prevention, $C T$ computed tomography, FiO ${ }_{2}$ inspired oxygen fraction, ICNARC intensive care national audit and research centre, ICU intensive care unit, MERS Middle Eastern Respiratory Syndrome, N/A not applicable, PaO ${ }_{2}$ arterial partial pressure of oxygen, $R R$ respiratory rate, $S A R S$ severe acute respiratory syndrome, $\mathrm{SpO}_{2}$ oxygen saturation

observation). Age and sex were key foci as potential risk factors, comparing patients with COVID-19 to either: a) SARS-CoV or Middle Eastern Respiratory Syndrome (MERS), or b) other forms of pneumonia. Generally, sex ratios were skewed such that men were over-represented among those with disease. In England, Northern Ireland, and Wales, Asian and Black individuals were found to be at increased risk of COVID-19 in descriptive analyses, with 15.4 and $10.7 \%$ of patients falling into these groupings, respectively, versus 5.8 and $2.8 \%$ of individuals with other viral pneumonia [30]. Higher body mass index (BMI) was also suggested to be a risk factor with two descriptive analyses, for example in the Intensive Care National Audit and Research Centre (ICNARC) report $31.2 \%$ of COVID-19 patients had a BMI of $30-<40$, versus $23.5 \%$ of people with other viral pneumonia [30, 37]. Given the large, national, scope of the ICNARC dataset, results from it are particularly likely to be reliable.

\section{Risk factors for severe disease}

Among the 20 studies of risk factors for severe versus milder disease and one of a mixed outcome (severe disease and death), a wide array of definitions of severity were used, such as ICU admission, the need for mechanical ventilation, and various measures of respiration and oxygenation (Table 2). Many risk factors were examined (Table 5). As well as potential demographic risks (age, sex, ethnicity), behavioural traits (smoking) and broad clinical factors (BMI, infectious diseases) were analysed. Large numbers of papers sought to explore the implications of different comorbidities on the risk of severe COVID-19, particularly respiratory and cardiovascular conditions.

The least equivocal evidence was presented for age as a risk factor, including four studies where it was an independent risk in a multivariable regression model $[19,20$, $31,36]$. The clearest analysis to present age data (i.e. which used different comparison groups) was a univariable regression model where individuals 65 years and over had 3.26 times the hazard rate of ARDS than those 


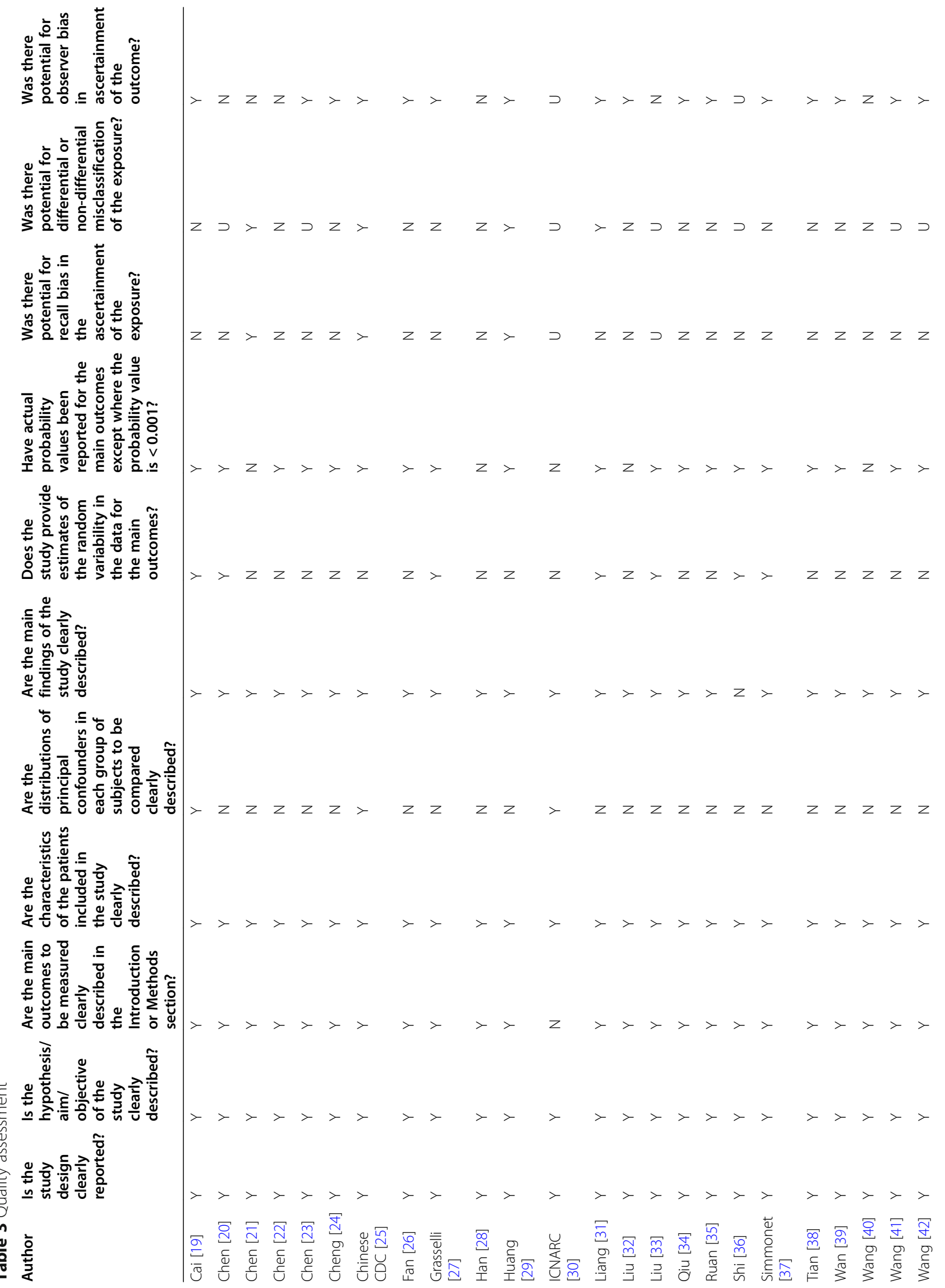




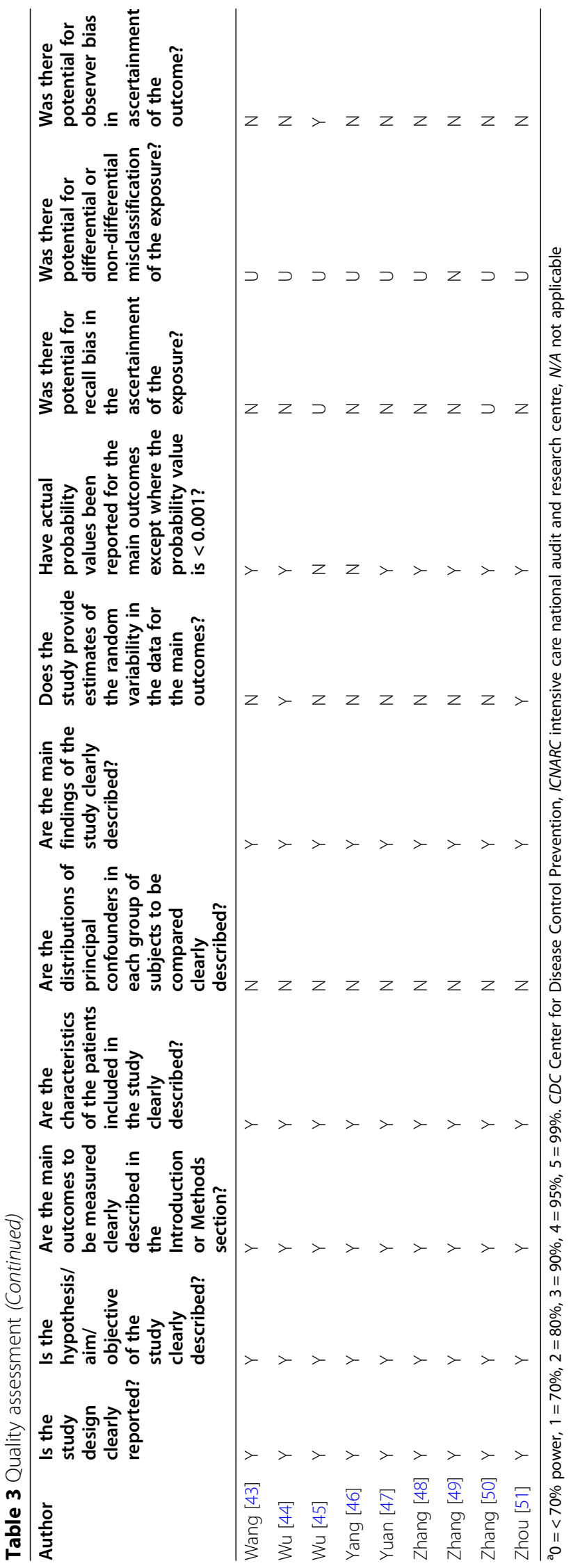




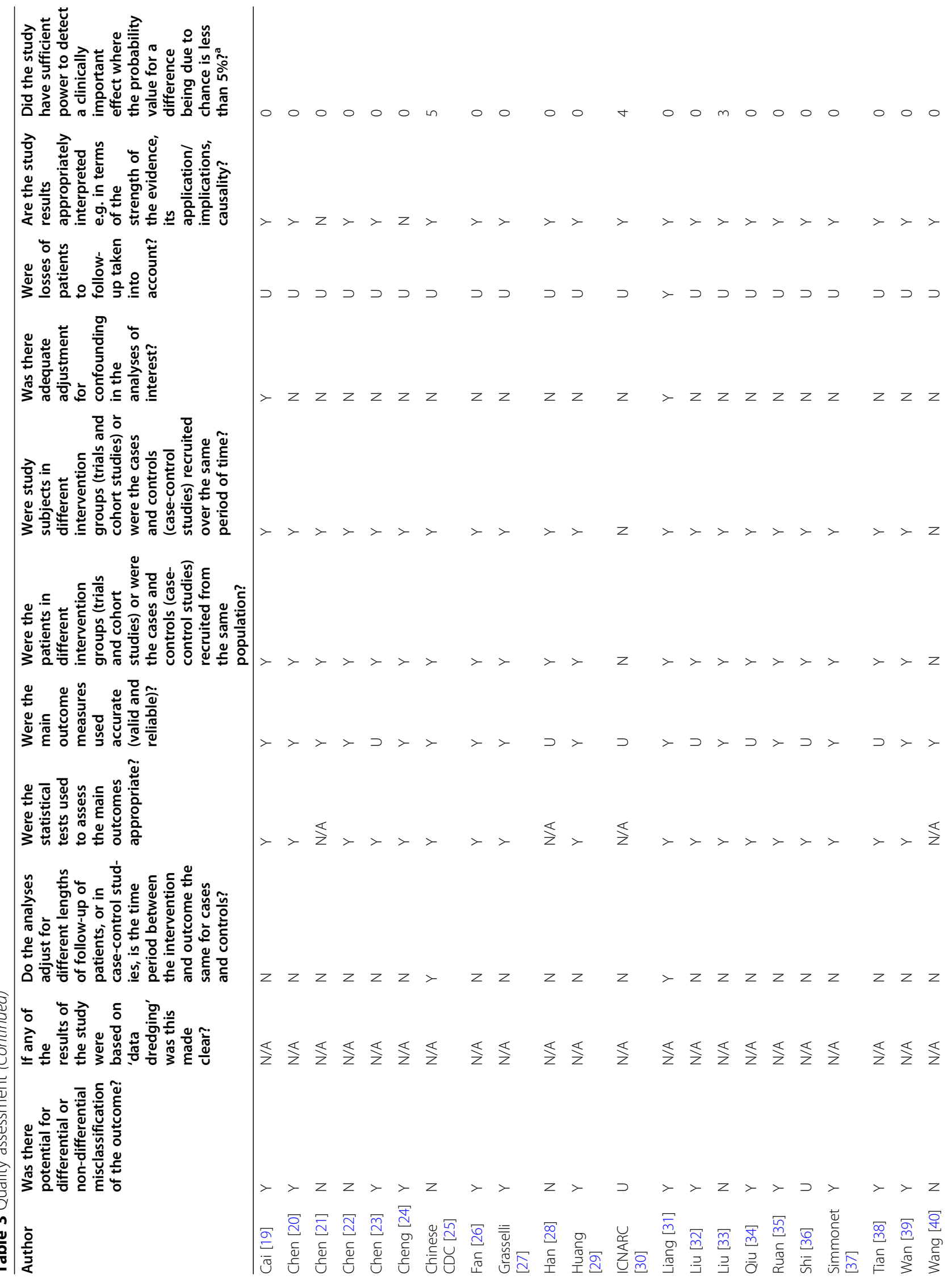




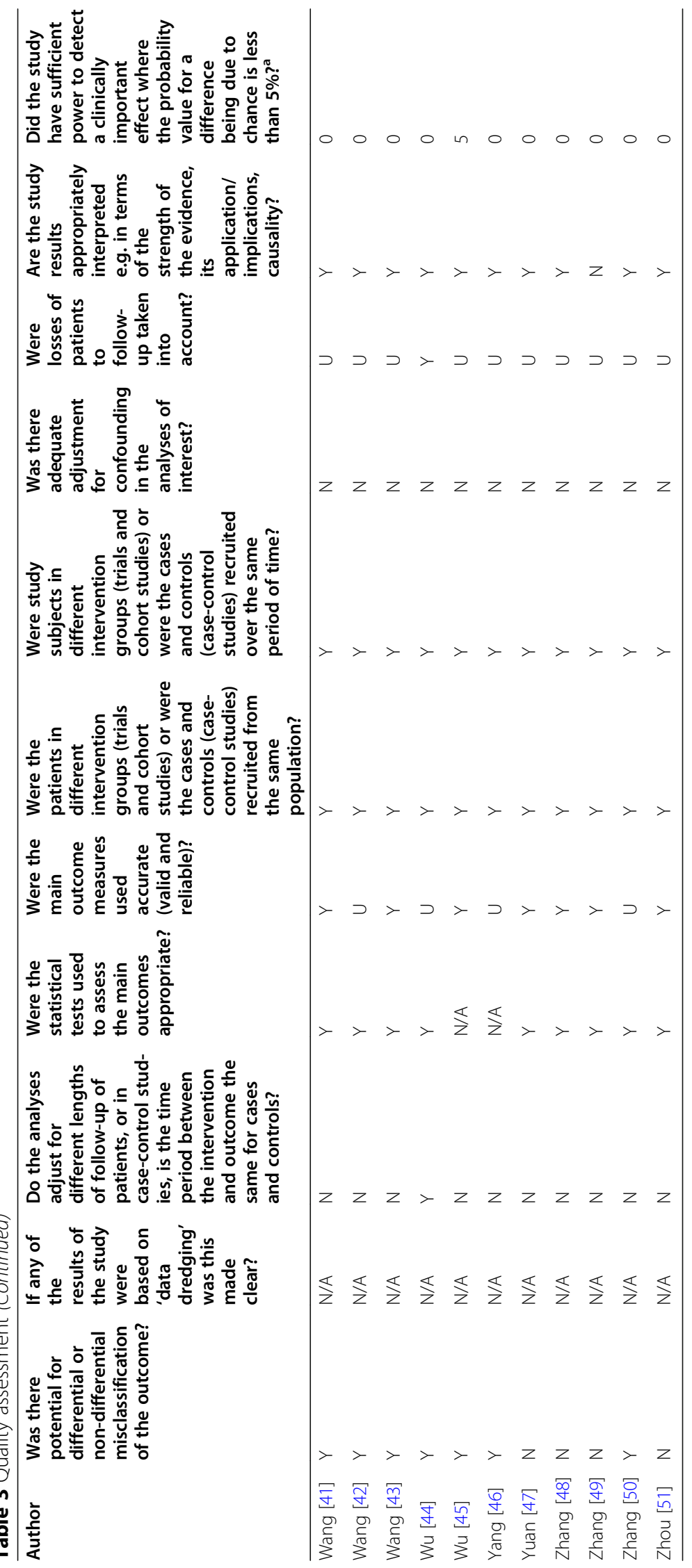


Table 4 Potential risk factors for disease

\begin{tabular}{|c|c|c|}
\hline $\begin{array}{l}\text { Potential risk } \\
\text { factor }\end{array}$ & Study supports risk & Study does not support risk or is neutral \\
\hline$\overline{\text { Age }}$ & $\begin{array}{l}\text { Patients older than those with COVID-19 and younger than those with } \\
\text { MERS (descriptive) [40] } \\
\text { Younger ages (median } 45 \text { years) in patients with COVID-19 than other } \\
\text { pneumonia (median 61) (statistical test) [22] } \\
\text { Increasing risk of positivity for SARS-CoV-2 among COVID-19 suspects (on } \\
\text { the basis of symptoms/contact tracing) with age (odds ratio 1.02) but un } \\
\text { clear categorisation of age (multivariable regression) [33] }\end{array}$ & $\begin{array}{l}\text { Median age } 60 \text { years in those with COVID-19 and } 61 \text { in } \\
\text { those with other viral pneumonia (descriptive) [30] } \\
\text { Mean age } 50 \text { years in COVID-19 patients vs. } \\
44 \text { in individuals with other pneumonia (statistical test) [24. }\end{array}$ \\
\hline Sex & $\begin{array}{l}\text { Sex ratio skewed towards men for COVID-19, akin to MERS but not SARS } \\
\text { (descriptive) [40] } \\
\text { Sex ratio skewed towards men for COVID-19 versus other viral pneumonia } \\
\text { (descriptive) [30] } \\
\text { Greater proportion male in COVID-19 versus other pneumonia, although } \\
\text { small sample size and thus low statistical certainty (statistical test) [24] } \\
\text { Increasing risk of positivity for SARS-CoV-2 among COVID-19 suspects (on } \\
\text { the basis of symptoms/contact tracing) among males versus female (odds } \\
\text { ratio 1.16) (logistic regression) [33] }\end{array}$ & $\begin{array}{l}\text { Sex distribution similar amongst patients } \\
\text { with COVID-19 and other pneumonia (statis } \\
\text { tical test) [22] }\end{array}$ \\
\hline Ethnicity & $\begin{array}{l}\text { Higher percentage of Black and Asian individuals amongst COVID-19 pa } \\
\text { tients than patients with other viral pneumonias (descriptive) [30] }\end{array}$ & \\
\hline $\begin{array}{l}\text { Index of multiple } \\
\text { deprivation }\end{array}$ & & $\begin{array}{l}\text { Distribution of deprivation similar across } \\
\text { COVID-19 and other viral pneumonia (de } \\
\text { scriptive) [30] }\end{array}$ \\
\hline $\begin{array}{l}\text { Body mass } \\
\text { index }\end{array}$ & $\begin{array}{l}\text { Greater proportion of COVID-19 patients had higher body mass index than } \\
\text { individuals with other pneumonia (descriptive) [37] } \\
\text { Greater proportion of COVID-19 patients had higher body mass index than } \\
\text { individuals with other viral pneumonia (descriptive) [30] }\end{array}$ & \\
\hline Pregnancy & & $\begin{array}{l}\text { Percentage of women who were pregnant } \\
\text { similar across COVID-19 and other viral pneu } \\
\text { monia (descriptive) [30] }\end{array}$ \\
\hline
\end{tabular}

MERS middle eastern respiratory syndrome, SARS severe acute respiratory syndrome

under 65 [44]. Eight studies suggested that diabetes could be a risk factor $[19,31,36,39,41,43,44,50]$, six hypertension [31, 36, 41, 43, 44, 50], and four the presence of unspecified comorbidities) [39, 41, 48, 50], but the balance of evidence for these co-morbidities being risk factors was generally inconclusive. Many other factors were examined by one study, often with small numbers of individuals with the condition. None of the included studies for disease severity were assessed to have been powered to detect a $10 \%$ increase in effect size.

\section{Risk factors for mortality}

Ten studies examined risk factors for mortality, often by nesting case-control studies within prospective or retrospective cohorts (Table 6). Among these studies, many included statistical testing, but none presented an adjusted regression model for the risk factors considered.

Eight studies examined age and all provided evidence for it being a risk factor for mortality $[21,25,27,35,44-$ 47], although none adjusted for other factors, such as comorbidities. Age groups from 50 upwards were considered particularly at risk. In the single regression analysis, the hazard rate for death in those 65 years or over was estimated to be six times that of individuals under 65 [44]. The evidence was similarly consistent for general comorbidities (albeit all the studies were descriptive); among individuals who died, comorbidities were 1.5 to 2.8 times more common than among those who survived [21, 35, $46,47,51]$. Specific comorbidities were discussed in several studies, generally under overarching classifications such as 'cardiovascular disease' or 'diabetes', with more specific definitions not provided. Evidence was more equivocal, but still in favour, of hypertension [3, 21, 25, 27, $47,51]$, cardiovascular disease $[21,25,35,45,47,51]$, diabetes $[21,25,45-47,51]$, and chronic respiratory/lung diseases being risk factors (references presented for studies in support only) $[21,45,51]$. Of these studies, data from two well-powered, national-level studies from China supported cardiovascular disease and diabetes as risk factors for mortality from COVID-19 [25, 45].

\section{Discussion}

In this systematic review of risk factors for COVID-19 disease, disease severity and mortality, we document 33 comparative studies examining sociodemographic, behavioural and clinical exposures. Age and sex were very commonly examined; a wide array of comorbidities have also been considered.

Within the synthesised evidence, risk factors for mortality were the clearest, plausibly partly because this outcome is easy to define. Increasing age (different studies presented different thresholds, but being over 50 years of age was common) was an uncontested risk factor. Five 
Table 5 Potential risk factors for disease severity

\begin{tabular}{lll}
\hline Potential risk factor & Study supports risk & Study does not support risk or is neutral \\
\hline Sex & Odds ratio for severe disease 3.68 for men compared to & $\begin{array}{l}\text { Sex distribution similar in severe and non-severe disease } \\
\text { (descriptive) [30, 49] }\end{array}$ \\
& women (multivariable regression) [36] & Odds ratio for invasive mechanical ventilation 2.83 for men \\
& Compared to women (multivariable regression) [37] & (statistical test) [23, 26, 28, 29, 32, 34, 38, 39, 41-44, 48-50] \\
& $\begin{array}{l}\text { Females less likely to be admitted to ICU, require } \\
\text { mechanical ventilation, or die; odds ratio 0.61 (multivariable }\end{array}$ & $\begin{array}{l}\text { Sex distribution similar in severe and non-severe disease } \\
\text { (multivariable regression) [19, 20] }\end{array}$
\end{tabular}

Age

Ethnicity

Deprivation

Pregnancy

Smoking

Body mass index

Any/other comorbidity logistic regression) [31]

Average $^{\text {a }} 61$ years severe disease, 45 otherwise (statistical test) [38]

Average $^{\mathrm{a}} 61$ years severe disease, 52 years moderate disease (statistical test) [23]

Average $^{a} 56$ years severe disease, 44 years mild disease (statistical test) [39]

Median 67 years acute respiratory distress syndrome, 52 severe, 45 mild (statistical test) [42]

Median 64 years severe patients, 52 years otherwise (statistical test) [48]

Mean 61 years severe, otherwise 40 (statistical test) [50]

Median 71 years $\mathrm{SpO}_{2}<90 \%, 37$ years $\mathrm{SpO}_{2} \geq 90 \%$ (statistical test) [43]

Median 66 years patients in ICU, 51 otherwise (statistical test) [41]

Median 54 years patients in ICU, 41 otherwise (statistical test) [26]

65 years and over 3.26 times the hazard rate of ARDS than those under 65 (univariable regression) [44] Age associated with ICU admission, odds ratio 1.06 but unclear for what categorisation of age (multivariable regression) [20]

Mean 56 years severe disease, 45 years mild disease; odds ratio 1.06 but unclear categorisation of age (multivariable regression) [36]

Mean 63 years severe disease, 41 years mild disease; odds ratio 1.08 but unclear categorisation of age (multivariable regression) [19]

Older individuals more likely to be admitted to ICU, require mechanical ventilation, or die; odds ratio 1.05, categories of age unclear (multivariable logistic regression) [31]

76.3\% of individuals receiving basic respiratory support were White versus $65.6 \%$ receiving advanced respiratory support; Asian and Black ethnicities appear most at risk of severe disease (England, Northern Ireland and Wales; descriptive) [30]

Distribution of disease severity similar across ethnic groups (Chinese, Malay, Indian, other - with small numbers in groups other than Chinese; study in Singapore; descriptive) [26]

Distribution across deprivation categories similar (descriptive) [30]

Distribution in pregnant and non-pregnant individuals similar across disease severity (descriptive) [30]

Distribution in current and non-current smokers similar across disease severity (descriptive), only three individuals smoked [39]

Distribution in current and non-current smokers similar across disease severity (statistical test); small numbers who smoked [29]

Distribution in historical/current and non-smokers similar across disease severity (statistical test) [36, 48]

Distribution of disease severity similar across body mass

$\geq 35 \mathrm{~kg} / \mathrm{m}^{2}$ risk factor versus $<25 \mathrm{~kg} / \mathrm{m}^{2}$ for invasive mechanical ventilation; odds ratio 7.36. Results for other strata cross the null (multivariable regression) [37]

Increasing body mass index increased risk; odds ratio 1.17 (categorisation unclear) [19]

Presence of comorbidity more common among those with severe disease (statistical test) $[39,41,48,50]$
Distribution with and without condition similar across disease severity (statistical test) $[23,29,36]$ Distribution with and without comorbidities not otherwise considered in the study similar across disease severity (statistical test) [50] 
Table 5 Potential risk factors for disease severity (Continued)

\begin{tabular}{|c|c|}
\hline Potential risk factor & Study supports risk \\
\hline $\begin{array}{l}\text { Cardiovascular disease/ } \\
\text { chronic heart disease/ } \\
\text { coronary heart disease }\end{array}$ & $\begin{array}{l}\text { Presence of comorbidity more common among those with } \\
\text { severe disease (descriptive) [39] } \\
\text { Presence of comorbidity more common among those with } \\
\text { severe disease (statistical test) }[19,36,41,43,50]\end{array}$ \\
\hline Hypertension & $\begin{array}{l}\text { Presence of comorbidity more common among those with } \\
\text { severe disease (statistical test) [41, 43, 50] } \\
\text { Hazard ratio of ARDS } 1.82 \text { in those with the condition versus } \\
\text { those without (univariable regression) [44] } \\
\text { Odds ratio of severe disease } 2.71 \text { in those with the condition } \\
\text { versus those without (multivariable regression) [36] } \\
\text { Odds ratio of being admitted to ICU, require mechanical } \\
\text { ventilation, or die } 1.89 \text { in those with the condition versus } \\
\text { those without (multivariable regression) [31] }\end{array}$ \\
\hline
\end{tabular}

Diabetes

Presence of comorbidity more common among those with severe disease (descriptive) [39]

Presence of comorbidity more common among those with severe disease (statistical test) $[19,36,41,43,50]$

Hazard ratio of ARDS 2.34 in those with the condition versus those without (univariable regression) [44]

Odds ratio of being admitted to ICU, require mechanical ventilation, or die 2.21 in those with the condition versus those without (multivariable regression) [31]

Respiratory/pulmonary

disease

Asthma

Chronic obstructive pulmonary disease (COPD)

Presence of comorbidity more common among those with severe disease (descriptive); small numbers with condition [39]

Presence of comorbidity more common among those with severe disease (statistical test); both studies have small numbers with the condition $[41,50]$

Odds ratio of being admitted to ICU, require mechanical ventilation, or die 3.40 in those with the condition versus those without (multivariable regression) [31]

Pulmonary tuberculosis

Malignancy

Cerebrovascular disease

Presence of comorbidity more common among those with severe disease (statistical test); small numbers with condition [39]

Presence of comorbidity more common among those with severe disease (statistical test) $[36,42,50]$

Presence of comorbidity more common among those with severe disease (multivariable analysis) [31]

Study does not support risk or is neutral

Distribution with and without condition similar across disease severity (multivariable regression) [20]

Distribution with and without condition similar across disease severity (descriptive) [30,44]

Distribution with and without condition similar across disease severity (statistical test) $[29,48]$

Distribution with and without condition similar across disease severity (descriptive) [39]

Distribution with and without condition similar across disease severity (statistical test); one study with small numbers with the condition $[23,29,42,48]$

Confidence interval in presence and absence of condition crosses the null (multivariable regression) [19]

Confidence interval in presence and absence of condition crosses the null (multivariable regression, result borderline) [37]

Distribution with and without condition similar across disease severity (statistical test); small numbers with condition [23]

Distribution with and without condition similar across disease severity (statistical test) $[29,48]$ Distribution with and without condition similar across disease severity (statistical test, borderline result) [42] Confidence interval in presence and absence of condition crosses the null (multivariable regression) [37]

Distribution with and without condition similar across disease severity (descriptive) [30, 39]

Distribution with and without condition similar across disease severity (statistical test); small numbers with condition [43]

Distribution with and without condition similar across disease severity (statistical test); small numbers with condition $[29,43,48]$

Distribution with and without condition similar across disease severity (statistical test); small numbers with condition [48]

Distribution with and without condition similar across disease severity (descriptive) [30]

Distribution with and without condition similar across disease severity (statistical test) [41]

Distribution with and without condition similar across disease severity (statistical test); small numbers with condition $[19,29,43]$

Presence of comorbidity more common among those with severe disease (statistical test) [41]

Arrhythmia

Cerebral infarction

Stroke

Distribution with and without condition similar across disease severity (statistical test); small numbers with the condition [48]

Distribution with and without condition similar across disease severity (statistical test) [42]

Distribution with and without condition similar across disease severity (statistical test); small numbers with condition [48]

Distribution with and without condition similar across disease severity (statistical test); small numbers with 
Table 5 Potential risk factors for disease severity (Continued)

\begin{tabular}{ll}
\hline Potential risk factor & Study supports risk \\
\hline $\begin{array}{ll}\text { Chronic kidney disease/ } \\
\text { renal issues }\end{array}$ & $\begin{array}{l}\text { Presence of comorbidity more common among those with } \\
\text { severe disease (statistical test) [42] }\end{array}$
\end{tabular}

\section{Study does not support risk or is neutral}

condition [48]

Distribution with and without condition similar across disease severity (descriptive) [30] Distribution with and without condition similar across disease severity (statistical test); small numbers with the condition [41]

Chronic renal disease/ insufficiency

Chronic liver disease

Fatty liver and abnormal liver function

Hyperlipidaemia

Dyslipidemia

Chronic gastritis/gastric

ulcer

Cholelithiasis

Urolithiasis

Thyroid diseases

Electrolyte imbalance

Presence of comorbidity more common among those with severe disease (statistical test); small numbers with condition [48]

Agglomerative disease

Immunocompromised

Chronic hepatitis

HIV

Living without assistance
Distribution with and without condition similar across disease severity (statistical test); one study has small numbers of patients with the condition $[36,48]$

Distribution with and without condition similar across disease severity (descriptive), sometimes small numbers with condition [19, 30, 39]

Distribution with and without condition similar across disease severity (statistical test) $[36,41]$

Distribution with and without condition similar across disease severity (statistical test); small numbers with condition $[29,50]$

Distribution with and without condition similar across disease severity (statistical test) [48]

Distribution with and without condition similar across disease severity (statistical test) [48]

Confidence interval in presence and absence of condition crosses the null (multivariable regression) [37]

Distribution with and without condition similar across disease severity (statistical test) [48]

Distribution with and without condition similar across disease severity (statistical test) [48]

Distribution with and without condition similar across disease severity (statistical test); small numbers with condition [48]

Distribution with and without condition similar across disease severity (statistical test); small numbers with the condition [48]

Distribution with and without condition similar across disease severity (descriptive); small numbers with the condition [39]

Distribution with and without condition similar across disease severity (descriptive) [30]

Distribution with and without condition similar across disease severity (statistical test); small numbers with condition [43]

Distribution with and without condition similar across disease severity (statistical test); small numbers with condition [41]

Distribution with and without condition similar across disease severity (descriptive) [30]

One study included death in a combined measure of disease severity [31]. ${ }^{\text {Undear }}$ as to whether mean, median or mode. ARDS acute respiratory distress syndrome, ICU intensive care unit, $\mathrm{SpO}_{2}$ oxygen saturation

studies also presented evidence for the presence of any comorbidities being a risk factor [21, 35, 46, 47, 51], with none demonstrating evidence against. Given the increasing prevalence of comorbidities with age, the lack of adjustment for confounding in these studies likely over- emphasises the effect size of each risk factor. We note that work subsequent to our literature search documents an independent effect of age on COVID-19 mortality from overall comorbidities, as measured by the Charlson Comorbidity Index Score, but not vice-versa [52]. 
Table 6 Potential risk factors for mortality

\begin{tabular}{ll}
\hline Potential risk factor & Study supports risk \\
\hline Sex & Men more at risk (descriptive) [21] \\
& \\
Age & Over 60 years particularly at risk (descriptive) [21] \\
& $8 \%$ case fatality ratio in 70-79year olds and 14.8\% in those \\
& over 80 . Overall figure 2.3\% (descriptive) [45] \\
& Median age in those who died 52 years, 65 years among \\
& survivors (descriptive) [46] \\
& Over 50 years of age particularly at risk- $1.3 \%$ died 50-59 \\
& years, 3.6\% 60-69years, 8.0\% 70-79years, 14.8\% 80 years \\
& plus; less than 1\% all other age groups (descriptive) [25] \\
& Risk begins to increase at approximately 50 years (statistical \\
& test, but graphical presentation) [35] \\
& Median age in those who died 68 years, among those who \\
& survived 55 (statistical test) [47] \\
& Over 61 years, increasing per 10 year age group (statistical \\
& test) [27] \\
& 65 years and older 6.17 the hazard rate of those under 65 \\
& (univariable regression) [44]
\end{tabular}

Smoking

\section{Study does not support risk or is neutral}

Sex distribution similar amongst patients who died and survived (descriptive) [25, 35, 46, 47]

Confidence interval for males versus females crosses the null (univariable regression) [44]

Proportion of smokers similar among those who died versus those who did not (descriptive); one study had small numbers of smokers $[21,46]$

Distribution of current smokers similar among survivors and non-survivors (univariable regression analysis, not included in multivariable model) [51]

Proportion of women who were pregnant similar amongst patients who died versus survived (descriptive) [21]

Any comorbidity

Presence of any comorbidity more common among those dying (descriptive) [21, 35, 46, 47, 51]

Hypertension

Presence of condition more common among those dying (descriptive) $[3,21,25,27]$

Presence of condition more common among those dying (statistical test) $[47,51]$

Cardiovascular disease/ Presence of condition more common among those dying chronic heart disease (descriptive) $[21,25,35,45]$

Presence of condition more common among those dying (statistical test) $[47,51]$

Diabetes

Presence of condition more common among those dying (descriptive) $[21,25,45,46]$

Presence of condition more common among those dying (statistical test) $[47,51]$

Chronic respiratory/lung Presence of condition more common among those dying disease (chronic (descriptive) $[21,45]$

obstructive lung disease) Presence of condition more common among those dying (statistical test) [51]

Respiratory infectious Presence of condition more common among those dying disease

Malignancy (descriptive) [25]

Presence of condition more common among those dying (descriptive) $[3,25]$

Cerebral infarction/ cerebrovascular disease

Presence of condition more common among those dying (descriptive) [46]

Chronic gastritis

Chronic kidney disease
Confidence interval for individuals with and without the condition crosses the null (univariable regression) [44]

Distribution dying in presence and absence of comorbidity similar (descriptive), sometimes small numbers with the condition $[44,46]$

Confidence interval for individuals with and without the condition crosses the null (univariable regression) [44]

Distribution dying in presence and absence of comorbidity similar (descriptive) [46]

Distribution dying in presence and absence of comorbidity similar (descriptive), sometimes small numbers with the condition $[46,47,51]$

Distribution dying in presence and absence of comorbidity similar (statistical test); small numbers with the condition [47]

Distribution dying in presence and absence of comorbidity similar (statistical test); small numbers with the condition [47]
Presence of condition more common among those dying (statistical test); small numbers with the condition [51] 
Table 6 Potential risk factors for mortality (Continued)

\begin{tabular}{|c|c|c|}
\hline Potential risk factor & Study supports risk & Study does not support risk or is neutral \\
\hline Dementia & & $\begin{array}{l}\text { Distribution dying in presence and absence of comorbidity } \\
\text { similar (descriptive); small numbers with the condition [46] }\end{array}$ \\
\hline Malnutrition & & $\begin{array}{l}\text { Distribution dying in presence and absence of comorbidity } \\
\text { similar (descriptive); small numbers with the condition [46] }\end{array}$ \\
\hline $\begin{array}{l}\text { Hepatitis B virus } \\
\text { infection }\end{array}$ & & $\begin{array}{l}\text { Distribution dying in presence and absence of comorbidity } \\
\text { similar (descriptive) [21] }\end{array}$ \\
\hline
\end{tabular}

Another study published outside of the time range of our search found both age and an array of comorbidities, each analysed separately (chronic cardiac disease, chronic pulmonary disease, chronic kidney disease, chronic neurological disease, dementia, malignancy, moderate/severe liver disease; and obesity), to be independent risk factors (as well as sex) [53].

Risk factors for severe disease were more complex to synthesise, likely due to the mixed array of outcome measures that can also be prone to observer bias. The impact of age was very commonly assessed, generally showing evidence in favour of this being a risk factor (with a similar age spectrum to the mortality data). Ethnicity was studied in two publications internationally $[26,30]$, with mixed results. We note that such findings are likely to be highly context-specific, given that ethnicity acts as a proxy for a series of sociodemographic factors that are highly relevant to the spread of an infectious condition (as well as, perhaps, some biological traits).

Studies of risk factors for COVID-19 disease have been complicated by testing strategies globally, which have largely been concentrated on severe disease. As our knowledge of the full symptom spectrum of the disease moves forward, it will be possible to have a broader case definition that does not solely focus on viral testing, and thus the ability for more generalised complementary studies. Additionally, serological surveys assessing the history of infection with SARS-CoV-2 in different population groups will allow the identification of risk factors for infection, whether symptomatic or not. Both ethnicity (Black and Asian individuals at higher risk; from a single study in England, Northern Ireland and Wales) [30] and higher BMI were found to be associated with disease severity within the included literature [30, 37], again from descriptive studies only. While these studies were not eligible for our review, we note a series of reports from non-comparative studies documenting the potential influence of ethnicity on the likelihood of getting COVID-19 e.g. the work of Price-Haywood from the US [52]. Male sex was reasonably consistently shown to be a risk factor for presence of COVID-19 but not with severity of disease or mortality $[24,30,40]$. As with ethnicity, socioeconomic and behavioural factors make this association likely to vary between settings.
In considering the role of comorbidities in COVID-19, it is important to consider the underlying pathology of the virus. Respiratory coronaviruses associated with the common cold in immunocompetent people generally affect only cells in the upper respiratory tract (URT), whereas the previously discovered highly pathogenic coronaviruses SARS-CoV and MERS-CoV affect cells in the URT and lower respiratory tract (LRT). SARS-CoV-2 has been shown to do the same [54], and one of the host cell receptors it targets is Angiotensin-Converting Enzyme 2 (ACE2), with a second major receptor being Transmembrane Serine Protease 2 (TMPRSS2) [55]. SARS-CoV-2 can infect all the major cell types in the respiratory tract - type I and type II pneumocytes, alveolar macrophages and endothelial cells $[56,57]$. This infection leads to cell death, with significant leaking of fluid into the alveolar spaces (pulmonary oedema), which compromises gas exchange [58], eventually leading to ARDS. The inflammatory response adds aggregation of repair proteins such as fibrin, which can lead to creation of hyaline membranes which further reduces the surface available for gas exchange [58]. Subsequently, inflammatory cells are activated, recruited by release or exposure of cytokines such as the interleukins (IL) $1 \beta$ and 6 , monocyte chemoattractant protein-1 [56], and proteins of the extracellular matrix, as well as upregulation of the complement system. Inflammatory cells release cytokines which have systemic effects, eventually leading to disseminated intravascular coagulation (DIC), hypotensive shock and metabolic disturbances if not checked [58].

This pathogenesis therefore offers several points where co-morbidities may exacerbate the process. The target receptor TMPRSS2 is modulated in response to air pollution and in autoimmune conditions such as asthma [55], which may affect the number of receptors available for SARS-CoV-2 to target, and ACE2 is involved in the renin-angiotensin system (RAS) which controls blood pressure. Viral interference causes dysfunction, which leads to a pro-inflammatory state and increased vascular permeability in response to changes in vascular contraction and sodium homeostasis - exacerbating the effect from the physical damage to the affected cells [58]. Conditions causing hypertension - both primary and secondary to renal disease, endocrine dysfunctions such 
as hypothyroidism, cardiovascular dysfunction such as arteriosclerosis, or neurological dysfunctions such as acute stress - also affect the RAS [58], meaning that these conditions might be expected to exacerbate pathology caused by SARS-CoV-2. Any condition creating a pro-inflammatory state, such as type II diabetes or preexisting infection, or involving autoimmunity, such as type I diabetes, might also be expected to contribute to increased pathology. There is also the direct effect of cell damage - if the target tissues are already damaged this reduces 'spare' capacity and therefore the leeway for adaptation to allow the host to continue to maintain homeostasis whilst still being able to eliminate the pathogen and repair the damage. The need for inflammatory cells to clear the infection is also a potential area of interface with comorbidities e.g. conditions such as unsuppressed HIV infection, or congenital deficiencies, or cancer malignancies; or the administration of immunosuppressant drugs such as chemotherapy for cancer or steroids.

The effect of ageing was particularly strong within our review, both in terms of the magnitude of effect estimates and the number of studies presenting evidence. As well as the above impact of comorbidities, we note that the host's age may influence pathogenesis, both in terms of the likelihood of having various comorbidities, and also due to its effect on the immune system. Indeed, the immune system becomes less effective over time (immunosenescence), which affects the quality and number of immune system cells generated [59]. Given the scale of the impact of age documented within this review, it seems unlikely that its effect can be explained by a single or a small number of comorbidities which are yet to be detected. This opens up the need to explore biological markers, for example ACE2 [60], and markers of immunosenescence.

The strengths of our review include its systematic approach and broad use of search terms to avoid missing studies. We additionally present a quality assessment to aid the interpretation of the strength of the evidence. In some instances, included publications may have focussed on one specific outcome, whereas our quality assessment took the perspective of the outcomes extracted for this review. We were unable to detect instances where two publications used the same patient populations for their analyses, potentially over-emphasising certain findings. Given the global nature of the pandemic, our review includes studies from around the world, albeit with a large preponderance from China, including studies conducted early after the emergence of SARS-CoV-2 when the atrisk population was predominantly those who had contact with Huanan seafood market and their contacts, and not necessarily representative of the general population. We note a particular lack of studies from the
African continent and the Americas, which may have implications for generalisability. Given the rapidly evolving literature on COVID-19, we also note our exclusion of studies published online after April 2020 (and the time period in which the surrounding text was written), for example the Dai report on cancer as a risk factor [61] and our exclusion of preprints (which was undertaken to ensure that all included studies had undergone an external quality assessment prior to inclusion).

Across the included publications, variability in study design, exposure and outcome measurement, and analyses made exact syntheses of effect sizes across different risk factors very difficult. Measures of disease severity varied, e.g. admission to ICUs or clinical parameters such as percentage oxygen saturation of the blood. Even measures such as admission to ICU can be subjective and may be time-, clinician-, and health systemsdependent. If severity is recorded at admission, risk factors may reflect issues associated with delayed access to healthcare, which may differ between settings and healthcare systems. It is also important to note that, in some studies of disease severity, mild disease included both people who were hospitalised with symptoms and asymptomatic individuals identified through contact tracing. Generally, analyses were descriptive or univariable and thus did not control for confounding. As documented above, this may be particularly problematic when it comes to separating the impact of age and the presence of comorbidities, as well as for identifying which comorbidities truly increase risk, given that many patients may have multi-morbidity.

The implications of our findings are two-fold for COVID-19, firstly for current public health practice and secondly for the design of future studies. We flag a number of factors of interest that should be considered by governments and public health agencies when designing shielding strategies and the targeting of future vaccines, as well as in mathematical modelling projecting the likely impact of the pandemic over time. We note, however, the need for sensitive handling of population groups deemed to be at higher risk, and how such labelling does not devolve responsibility from public bodies to these individuals for their own welfare [8]. Some public health agencies are now including reporting of potential risk factors in their routine outputs, including ICNA $\mathrm{RC}$ (included in this review) [30] and the newer European Centre for Disease Prevention and Control reports, which were released after this review was conducted [62].

Our review demonstrates both the volume of literature that can be published within only a few months since the appearance of an emerging infectious disease, and the need for co-ordinated approaches to such pathogens. Global efforts using national datasets are hugely valuable 
in systematically determining the aetiology of a disease, particularly to detect smaller effect sizes. Determination of the exact threshold of important risk depends on public perceptions of the disease [63], as well as policy needs. Data collection should be standardised where possible, e.g. by using consistent definitions of outcomes and the treatment of exposures (for example for hypertension, given that blood pressure is continuous). (For COVID-19 we note both the valuable World Health Organization interim guidelines on its management in providing consistent approaches for testing and the definition of ARDS [14], and that platforms such as the International Severe Acute Respiratory and Emerging Infections Consortium (ISARIC) have aimed to facilitate such standardisation [64].) The choice of comparison groups should also merit careful consideration; comparison to other forms of the same condition (e.g. SARS and MERS for COVID-19), although interesting, provide little information about risk groups to be currently acted upon. Where key potential risk factors of interest, such as deprivation, are linked to both the disease of interest and the comparator condition, this limits the inferences possible. Saying this, studies of COVID-19 with the comparator group of other forms of viral pneumonia are a useful complement to studies using a general population comparator, as they show whether people with particular risk factors are at risk over and above what they might experience from 'normal' respiratory viruses, which might inform the level of additional precautions they could consider taking.

Finally, appropriately adjusted multivariable analyses should be prioritised, in order to separate the implications of different risk factors and to infer true causal relationships, for example exploring specific markers of comorbidity severity and control, such as the use of specific medications. We can then make the recommendations for shielding criteria more targeted, meaning that the public can be made more aware of the risk factors that are likely to have clinical significance and adapt their behaviour accordingly. Early clinical studies during pandemics are critically important and published rapidly under extremely difficult circumstances, but we would argue that high-quality epidemiological studies should also be seen as a priority, and that emergency response plans should include provision of appropriate epidemiological and statistical expertise.

\section{Conclusions}

The volume of literature generated in the short time since the appearance of SARS-CoV-2 has been considerable. Many studies have sought to document the risk factors for COVID-19 disease, disease severity and mortality. Age was the only risk factor based on robust studies and with a consistent body of evidence. Mechanistic studies are required to understand why age is such an important risk factor. At the start of pandemics, large, standardised, studies using multivariable analyses - e.g. using national surveillance data - are urgently needed in order to inform stratified approaches to rapidly protecting the population groups most at risk.

\begin{abstract}
Abbreviations
ACE2: Angiotensin-converting enzyme 2; ARDS: Acute respiratory distress syndrome; BMl: Body mass index; CDC: Center for Disease Control and Prevention; COPD: Chronic obstructive pulmonary disease; COVID19: Coronavirus disease-19; CT: Computed tomography; DIC: Disseminated intravascular coagulation; $\mathrm{FiO}_{2}$ : Inspired oxygen fraction; ICNARC: Intensive care national audit and research centre; ICU: Intensive care unit;

IL: Interleukin; ISARIC: International severe acute respiratory and emerging infections consortium; LRT: Lower respiratory tract; MERS: Middle Eastern Respiratory Syndrome; MS: Multiple sclerosis; N/A: Not applicable; $\mathrm{PaO}_{2}$ : Arterial partial pressure of oxygen; PCR: Polymerase chain reaction; RAS: Renin-angiotensin system; RR: Respiratory rate; SARS: Severe acute respiratory syndrome; SARS-CoV-2: Severe acute respiratory syndrome coronavirus-2; $\mathrm{SpO}_{2}$ : Oxygen saturation; TMPRSS2: Transmembrane serine protease 2; URT: Upper respiratory tract
\end{abstract}

\section{Acknowledgements}

Not applicable.

\section{Authors' contributions}

HRS, MF and AS conceived of the work. HRS, MF, CJ, EV and CS designed the work. MF, CJ, HRS, EV, MDM, YJ and XW acquired and interpreted the data. CRS, UA, CM, JLKM, LDR, CR, SJS, MEJW and AS also interpreted the data. HRS and MF drafted the work. CJ, EV, CRS, MDM, UA, CM, YJ, JLKM, LDR, CR, SJS, $X W, M E J W$ and $A S$ revised it critically for important intellectual content. All authors give final approval of the version to be published. All authors agree to be accountable for all aspects of the work in ensuring that questions related to the accuracy or integrity of any part of the work are appropriately investigated and resolved.

\section{Funding}

HRS and MF are supported by the Medical Research Council [MR/R008345/1] CJ's salary came through MRC core funding MC_UU_12023/26. SJS is funded by the Wellcome Trust [WT 209560/Z/17/Z]. CRS has received funding from the Medical Research Council [MR/R008345/1], the National Institute for Health Research [11/46/23] and the New Zealand Health Research Council [20/1018] and Ministry for Business, Innovation and Employment. EV is funded by the Medical Research Council [MR/R008345/1] through the EAVE II grant and supported by the Scottish Government. We also acknowledge the support of HDR UK. The views and opinions expressed here are those of the authors and do not necessarily reflect those of the Health Technology Assessment programme, NIHR, NHS, or the UK Department of Health. Our funders had no role in the design of the study and collection, analysis, and interpretation of data, and in writing the manuscript.

Availability of data and materials

All data generated or analysed during this study are included in this published article and the underlying papers.

\section{Declarations}

Ethics approval and consent to participate

This is a systematic review and therefore presents secondary data. No ethical approvals were required.

Consent for publication

Not applicable.

\section{Competing interests}

CR is a member of the Scottish Government's Chief Medical Officer's COVID19 Advisory Group, a member of the UK SPI-M committee and the 
Commission Human Medicines COVID-19 Vaccine Safety Benefits and Risks Working Group. JLKM is Incident Director for COVID-19 at Public Health Scotland and reports no conflicts of interest. LDR serves on a number of Scottish Government Advisory Groups, including COVID-19. MEJW is a member of the SPI-M advisory committee for the UK Government and the Covid19 Advisory Group for the Scottish Government. AS is a member of the Scottish Government's Chief Medical Officer's COVID-19 Advisory Group and the New and Emerging Respiratory Virus Threats (NERVTAG) Risk Stratification Subgroup. HRS is an advisor to the Scottish Parliament's COVID committee. The views represented in this article do not represent the views of the UK or Scottish Government. All other authors declare that they have no competing interests.

\section{Author details}

'Usher Institute, University of Edinburgh, 30 West Richmond Street Edinburgh EH8 9DX, UK. ${ }^{2}$ Medical Research Council Clinical Trials Unit, University College London, London, UK. ${ }^{3}$ School of Health, Wellington Faculty of Health, Victoria University of Wellington, Wellington, New Zealand. ${ }^{4}$ School of Medicine, University of St. Andrews, St. Andrews, UK. ${ }^{5}$ Freelance consultant, Beijing, People's Republic of China. ${ }^{6}$ National Health Service Fife, Kirkcaldy, UK. ${ }^{7}$ Public Health Scotland, Glasgow, UK. ${ }^{8}$ School of Medicine and Dentistry, University of Aberdeen, Aberdeen, UK. ${ }^{9}$ Department of Mathematics and Statistics, University of Strathclyde, Glasgow, UK. ${ }^{10}$ School of Biological Sciences, University of Edinburgh, Edinburgh, UK.

\section{Received: 17 June 2020 Accepted: 16 March 2021} Published online: 12 April 2021

\section{References}

1. World Health Organization. WHO Director-General's opening remarks at the media briefing on COVID-19 2020 [updated 11th March 2020. Available from: https://www.who.int/dg/speeches/detail/who-director-general-s-opening-rema rks-at-the-media-briefing-on-covid-19\%2D\%2D-11-march-2020. Accessed 20 Mar 2020.

2. CDC COVID-19 Response Team. Severe Outcomes Among Patients with Coronavirus Disease 2019 (COVID-19)- United States, February 12-March 16, 2020. Morb Mortal Wkly Rep. 2020;69(12):343-6.

3. Wu JT, Leung K, Bushman M, Kishore N, Niehus R, de Salazar PM, et al. Estimating clinical severity of COVID-19 from the transmission dynamics in Wuhan, China. Nat Med. 2020;26:506-10.

4. Ortiz JR, Perut $M$, Dumolard L, Wijesinghe PR, Jorgensen P, Ropero AM, Danovaro-Holliday MC, Heffelfinger JD, Tevi-Benissan C, Teleb NA, Lambach $P$, Hombach J. A global review of national influenza immunization policies: analysis of the 2014 WHO/UNICEF joint reporting form on immunization. Vaccine. 2016;34(45):5400-5. https://doi.org/10.1016/j.vaccine.2016.07.045.

5. NHS Digital. Coronavirus (COVID-19): Shielded patients list 2020. Available from: https://digital.nhs.uk/coronavirus/shielded-patient-list. Accessed 27 May 2020.

6. Health Protection Surveillance Centre. Guidance for Vulnerable Groups 2020 Available from: https://www.hpsc.ie/a-z/respiratory/coronavirus/novelcorona virus/guidance/vulnerablegroupsguidance/. Accessed 3 June 2020.

7. van Bunnik BAD, Morgan ALK, Bessell P, Calder-Gerver G, Zhang F, Haynes S, et al. Segmentation and shielding of the most vulnerable members of the population as elements of an exit strategy from COVID-19 lockdown. MedRxiv. 2020. https://doi.org/10.1101/2020.05.04.20090597.

8. Ganguli-Mitra A, Young I, Engelmann L, Harper I, McCormack D, Marsland R, et al. Segmenting communities as public health strategy: a view from the social sciences and humanities. Wellcome Open Res. 2020;5:104. https://doi. org/10.12688/wellcomeopenres.15975.1.

9. NHS Digital. Statement for people who think they have inaccurately been sent communication about being vulnerable to coronavirus 2020 [updated 24th March 2020. Available from: https://digital.nhs.uk/news-and-events/la test-news/statement-on-coronavirus-communications. Accessed 26 Mar 2020.

10. Public Health England. Guidance on social distancing for everyone in the UK and protecting older people and vulnerable adults 2020 [updated 30th March 2020. Available from: https:/www.gov.uk/government/publications/ covid-19-guidance-on-social-distancing-and-for-vulnerable-people/guidanceon-social-distancing-for-everyone-in-the-uk-and-protecting-older-people-a nd-vulnerable-adults. Accessed 27 Apr 2020.
11. Cabinet Office. Staying alert and safe (social distancing) 2020 [Available from: https://www.gov.uk/government/publications/staying-alert-and-safesocial-distancing/staying-alert-and-safe-social-distancing\#protectingdifferent-groups-of-people. Accessed 28 May 2020.

12. Public Health Scotland. Search criteria for highest risk patients for shielding. Version 4.02020 [Available from: https://hpspubsrepo.blob.core.windows. net/hps-website/nss/3008/documents/1_covid-19-search-criteria-highestrisk-patients.pdf. Accessed 3 June 2020.

13. Zheng YY, Ma YT, Zhang JY, Xie X. COVID-19 and the cardiovascular system. Nat Rev Cardiol. 2020;17(5):259-60. https://doi.org/10.1038/s41 569-020-0360-5

14. World Health Organization. Clinical management of severe acute respiratory infection (SARI) when COVID-19 disease is suspected Interim guidance 2020 [updated 13th March 2020. Available from: https://www.who.int/publica tions-detail/clinical-management-of-severe-acute-respiratory-infection-whennovel-coronavirus-. Accessed 27 May 2020. (ncov)-infection-is-suspected.

15. Downs SH, Black N. The feasibility of creating a checklist for the assessment of the methodological quality both of randomised and non-randomised studies of health care interventions. J Epidemiol Community Health. 1998; 52(6):377-84. https://doi.org/10.1136/jech.52.6.377.

16. Deeks JJ, Dinnes J, D'Amico R, Sowden AJ, Sakarovitch C, Song F, et al. Evaluating non-randomised intervention studies. Health Technol Assess. 2003;7(27):iii-X, 1-173.

17. Arner TGS, G.G.; Friedman, R.; Lantinga, M.; Sangam, S.; Zubieta, J.C.; Sullivan, K.M.; Brendel, K.A.; Gao, Z.; Fontaine, N.; Shu, M.; Fuller, G.; Smith, D.C.; Nitschke, D.A.; Fagan, R.F.;. Epi info ${ }^{\mathrm{TM}}$, a database and statistics program for public health professionals. GA, USA: Centers for Disease Control and Prevention; 2011.

18. Westreich D, Greenland S. The table 2 fallacy: presenting and interpreting confounder and modifier coefficients. Am J Epidemiol. 2013;177(4):292-8. https://doi.org/10.1093/aje/kws412.

19. Cai Q, Huang D, Ou P, Yu H, Zhu Z, Xia Z, et al. COVID-19 in a designated infectious diseases hospital outside Hubei Province, China. Allergy. 2020; 75(7):1742-52. https://doi.org/10.1111/all.14309.

20. Chen J, Qi T, Liu L, Ling Y, Qian Z, Li T, Li F, Xu Q, Zhang Y, Xu S, Song Z, Zeng Y, Shen Y, Shi Y, Zhu T, Lu H. Clinical progression of patients with COVID-19 in Shanghai, China. J Infect. 2020;80(5):e1-6. https://doi.org/10.101 6/j.jinf.2020.03.004.

21. Chen T, Wu D, Chen H, Yan W, Yang D, Chen G, et al. Clinical characteristics of 113 deceased patients with coronavirus disease 2019: Retrospective study. The BMJ. 2020;368(no pagination).

22. Chen $X$, Yang Y, Huang M, Liu L, Zhang X, Xu J, et al. Differences between COVID-19 and suspected then confirmed SARS-CoV-2-negative pneumonia: a retrospective study from a single center. J Med Virol. 2020;92(9):1572-79. https://doi.org/10.1002/jmv.25810.

23. Chen G, Wu D, Guo W, Cao Y, Huang D, Wang H, Wang T, Zhang X, Chen H, Yu H, Zhang X, Zhang M, Wu S, Song J, Chen T, Han M, Li S, Luo X, Zhao J, Ning Q. Clinical and immunological features of severe and moderate coronavirus disease 2019. J Clin Invest. 2020;130(5):2620-9. https://doi.org/1 $0.1172 / \mathrm{JCl} 137244$.

24. Cheng Z, Lu Y, Cao Q, Qin L, Pan Z, Yan F, Yang W. Clinical features and chest CT manifestations of coronavirus disease 2019 (COVID-19) in a singlecenter study in Shanghai, China. AJR Am J Roentgenol. 2020;215(1):121-6. https://doi.org/10.2214/AJR.20.22959.

25. Epidemiology Working Group for Ncip Epidemic Response CCfDC, Prevention. The epidemiological characteristics of an outbreak of 2019 novel coronavirus diseases (COVID-19) in China. Zhonghua Liu Xing Bing Xue Za Zhi. 2020;41(2):145-51.

26. Fan BE, Chong VCL, Chan SSW, Lim GH, Lim KGE, Tan GB, et al. Hematologic parameters in patients with COVID-19 infection. Am J Hematol. 2020;95(6): E131-E134. https://doi.org/10.1002/ajh.25774.

27. Grasselli G, Zangrillo A, Zanella A, Antonelli M, Cabrini L, Castelli A, Cereda D, Coluccello A, Foti G, Fumagalli R, lotti G, Latronico N, Lorini L, Merler S, Natalini G, Piatti A, Ranieri MV, Scandroglio AM, Storti E, Cecconi M, Pesenti A, for the COVID-19 Lombardy ICU Network. Baseline characteristics and outcomes of 1591 patients infected with SARS-CoV-2 admitted to ICUs of the Lombardy region, Italy. JAMA. 2020;323(16):1574-81. https://doi.org/10.1 001/jama.2020.5394.

28. Han H, Xie L, Liu R, Yang J, Liu F, Wu K, et al. Analysis of heart injury laboratory parameters in 273 COVID-19 patients in one hospital in Wuhan, China. J Med Virol. 2020;92(7):819-23. https://doi.org/10.1002/jmv.25809. 
29. Huang C, Wang Y, Li X, Ren L, Zhao J, Hu Y, Zhang L, Fan G, Xu J, Gu X, Cheng Z, Yu T, Xia J, Wei Y, Wu W, Xie X, Yin W, Li H, Liu M, Xiao Y, Gao H, Guo L, Xie J, Wang G, Jiang R, Gao Z, Jin Q, Wang J, Cao B. Clinical features of patients infected with 2019 novel coronavirus in Wuhan, China. Lancet. 2020;395(10223):497-506. https://doi.org/10.1016/S0140-6736(20)30183-5.

30. Intensive Care National Audit and Research Centre. ICNARC report on COVID19 in critical care 2020 [Available from: https://www.google.com/url?sa=t\&rct= $j \& q=\&$ esr $c=s \&$ source $=$ web\& $c d=\& c a d=r j a \& u a c t=8 \&$ ved $=2$ ahUKEwjS4MK86NbpA hVPQ0EAHQ3dD5gQFjAAegQ|BhAB\&url=https\%3A\%2F\%2Fwww.icnarc.org\%2 FDataServices\%2FAttachments\%2FDownload\%2Fc5a62b13-6486-ea11-912500505601089b\&usg=AOvVaw3iovn0oEBhP6xPnV27l9HR. Accessed 21 May 2020.

31. Liang W, Guan W, Chen R, Wang W, Li J, Xu K, Li C, Ai Q, Lu W, Liang H, Li S, He J. Cancer patients in SARS-CoV-2 infection: a nationwide analysis in China. Lancet Oncol. 2020;21(3):335-7. https://doi.org/10.1016/S1470-2045(2 0)30096-6.

32. Liu R, Ma Q, Han H, Su H, Liu F, Wu K, et al. The value of urine biochemical parameters in the prediction of the severity of coronavirus disease 2019. Clin Chem Lab Med. 2020;58(7):1121-24. https://doi.org/10.1515/cclm-20200220 .

33. Liu R, Han H, Liu F, Lv Z, Wu K, Liu Y, Feng Y, Zhu C. Positive rate of RT-PCR detection of SARS-CoV-2 infection in 4880 cases from one hospital in Wuhan, China, from Jan to Feb 2020. Clin Chim Acta. 2020;505:172-5. https://doi.org/10.1016/j.cca.2020.03.009.

34. Qiu H, Wu J, Hong L, Luo Y, Song Q, Chen D. Clinical and epidemiological features of 36 children with coronavirus disease 2019 (COVID-19) in Zhejiang, China: an observational cohort study. Lancet Infect Dis. 2020;20(6): 689-96. https://doi.org/10.1016/S1473-3099(20)30198-5.

35. Ruan Q, Yang K, Wang W, Jiang L, Song J. Clinical predictors of mortality due to COVID-19 based on an analysis of data of 150 patients from Wuhan, China. Intensive Care Med. 2020;46(5):846-48. https://doi.org/10.1007/ s00134-020-05991-X

36. Shi $Y, Y u$ X, Zhao H, Wang H, Zhao R, Sheng J. Host susceptibility to severe COVID-19 and establishment of a host risk score: findings of 487 cases outside Wuhan. Crit Care. 2020;24(1):108. https://doi.org/10.1186/s13054-02 0-2833-7.

37. Simonnet A, Chetboun M, Poissy J, Raverdy V, Noulette J, Duhamel A, et al. High prevalence of obesity in severe acute respiratory syndrome coronavirus-2 (SARS-CoV-2) requiring invasive mechanical ventilation. Obesity. 2020;28(7):1195-99. https://doi.org/10.1002/oby.22831.

38. Tian S, Hu N, Lou J, Chen K, Kang X, Xiang Z, Chen H, Wang D, Liu N, Liu D, Chen G, Zhang Y, Li D, Li J, Lian H, Niu S, Zhang L, Zhang J. Characteristics of COVID-19 infection in Beijing. J Infect. 2020;80(4):401-6. https://doi.org/1 0.1016/j.jinf.2020.02.018.

39. Wan S, Xiang Y, Fang W, Zheng Y, Li B, Hu Y, Lang C, Huang D, Sun Q, Xiong Y, Huang X, LV J, Luo Y, Shen L, Yang H, Huang G, Yang R. Clinical features and treatment of COVID-19 patients in Northeast Chongqing. J Med Virol. 2020;92(7):797-806. https://doi.org/10.1002/jmv.25783.

40. Wang C, Horby PW, Hayden FG, Gao GF. A novel coronavirus outbreak of global health concern. Lancet. 2020;395(10223):470-3. https://doi.org/10.101 6/S0140-6736(20)30185-9.

41. Wang D, Hu B, Hu C, Zhu F, Liu X, Zhang J, et al. Clinical Characteristics of 138 Hospitalized Patients with 2019 Novel Coronavirus-Infected Pneumonia in Wuhan, China. JAMA. 1061;323(11):1061-9.

42. Wang L, Li X, Chen H, Yan S, Li D, Li Y, Gong Z. Coronavirus disease 19 infection does not result in acute kidney injury: an analysis of 116 hospitalized patients from Wuhan, China. Am J Nephrol. 2020;51(5):343-8. https://doi.org/10.1159/000507471.

43. Wang Z, Yang B, Li Q, Wen L, Zhang R. Clinical features of 69 cases with coronavirus disease 2019 in Wuhan, China. Clin Infect Dis. 2020;71(15):76977. https://doi.org/10.1093/cid/ciaa272.

44. Wu C, Chen X, Cai Y, Xia J, Zhou X, Xu S, et al. Risk factors associated with acute respiratory distress syndrome and death in patients with coronavirus disease 2019 pneumonia in Wuhan, China. JAMA Intern Med. 2020;180(7): 934-43. https://doi.org/10.1001/jamainternmed.2020.0994.

45. Wu Z, JM MG. Characteristics of and Important Lessons from the Coronavirus Disease 2019 (COVID-19) Outbreak in China: Summary of a Report of 72314 Cases from the Chinese Center for Disease Control and Prevention. JAMA. 1239;323(13):1239-42

46. Yang $X, Y u Y, X u$ J, Shu H, Xia J, Liu H, et al. Clinical course and outcomes of critically ill patients with SARS-CoV-2 pneumonia in Wuhan, China: a single- centered, retrospective, observational study. Lancet Respir Med. 2020;8(5): 475-81. https://doi.org/10.1016/S2213-2600(20)30079-5.

47. Yuan M, Yin W, Tao Z, Tan W, Hu Y. Association of radiologic findings with mortality of patients infected with 2019 novel coronavirus in Wuhan, China. Plos One. 2020;15(3):e0230548. https://doi.org/10.1371/journal.pone.0230548.

48. Zhang JJ, Dong X, Cao YY, Yuan YD, Yang YB, Yan YQ, et al. Clinical characteristics of 140 patients infected with SARS-CoV-2 in Wuhan, China. Allergy: Eur J Allergy Clin Immunol. 2020;75(7):1730-41. https://doi.org/1 0.1111/all.14238.

49. Zhang G, Zhang J, Wang B, Zhu X, Wang Q, Qiu S. Analysis of clinical characteristics and laboratory findings of 95 cases of 2019 novel coronavirus pneumonia in Wuhan, China: a retrospective analysis. Respir Res. 2020;21(1): 74. https://doi.org/10.1186/s12931-020-01338-8.

50. Zhang R, Ouyang H, Fu L, Wang S, Han J, Huang K, et al. CT features of SARS-CoV-2 pneumonia according to clinical presentation: a retrospective analysis of 120 consecutive patients from Wuhan city. Eur Radiol. 2020. epub ahead of print. https://doi.org/10.1007/s00330-020-06854-1.

51. Zhou F, Yu T, Du R, Fan G, Liu Y, Liu Z, et al. Clinical course and risk factors for mortality of adult inpatients with COVID-19 in Wuhan, China: a retrospective cohort study. Lancet. 1054;395(10229):1054-62.

52. Price-Haywood EG, Burton J, Fort D, Seoane L. Hospitalization and mortality among Black patients and white patients with Covid-19. N Engl J Med. 2020;382(26):2534-43. https://doi.org/10.1056/NEJMsa2011686.

53. Docherty AB, Harrison EM, Green CA, Hardwick HE, Pius R, Norman L, et al. Features of 20133 UK patients in hospital with covid-19 using the ISARIC WHO clinical characterisation protocol: prospective observational cohort study. BMJ. 2020;369:m1985.

54. Chen J. Pathogenicity and transmissibility of 2019-nCoV-A quick overview and comparison with other emerging viruses. Microbes Infect. 2020;22(2): 69-71. https://doi.org/10.1016/j.micinf.2020.01.004.

55. Gengler IW, J.C.; Speth, M.M.; Sedaghat A.R.;. Sinonasal pathophysiology of SARSCoV-2 and COVID-19: a systematic review of the current evidence. Laryngoscope Invest Otolaryngol. 2020;5(3):354-9. https://doi.org/10.1002/lio2.384.

56. Chu H, Chan JF, Wang Y, Yuen TT, Chai Y, Hou Y, et al. Comparative replication and immune activation profiles of SARS-CoV-2 and SARS-CoV in human lungs: an ex vivo study with implications for the pathogenesis of COVID-19. Clin Infect Dis. 2020;71(6):1400-9. https://doi.org/10.1093/cid/ ciaa410

57. Fu Y, Cheng Y, Wu Y. Understanding SARS-CoV-2-mediated inflammatory responses: from mechanisms to potential therapeutic tools. Virol Sin. 2020; 35(3):266-71. https://doi.org/10.1007/s12250-020-00207-4.

58. Kumar VA, A.K.; Aster, J.C.; . Robbins \& Cotran pathologic basis of disease, 9e (Robbins pathology) 2014. 1408 p.

59. Pangrazzi L, Meryk A, Naismith E, Koziel R, Lair J, Krismer M, Trieb K, Grubeck-Loebenstein B. "Inflamm-aging" influences immune cell survival factors in human bone marrow. Eur J Immunol. 2017;47(3):481-92. https:// doi.org/10.1002/eji.201646570.

60. Bunyavanich S, Do A, Vicencio A. Nasal gene expression of angiotensinconverting enzyme 2 in children and adults. JAMA. 2020;323(23):2427-9. https://doi.org/10.1001/jama.2020.8707.

61. Dai M, Liu D, Liu M, Zhou F, Li G, Chen Z, Zhang Z, You H, Wu M, Zheng Q, Xiong $Y$, Xiong $H$, Wang $C$, Chen $C$, Xiong F, Zhang $Y$, Peng $Y$, Ge S, Zhen B, Yu T, Wang L, Wang H, Liu Y, Chen Y, Mei J, Gao X, Li Z, Gan L, He C, Li Z, Shi Y, Qi Y, Yang J, Tenen DG, Chai L, Mucci LA, Santillana M, Cai H. Patients with Cancer appear more vulnerable to SARS-CoV-2: a multicenter study during the COVID-19 outbreak. Cancer Discov. 2020;10(6):783-91. https:// doi.org/10.1158/2159-8290.CD-20-0422.

62. European Centre for Disease Prevention and Control. ECDC launches new weekly COVID-19 surveillance report 2020 [Available from: https://www. ecdc.europa.eu/en/news-events/ecdc-launches-new-weekly-covid-19surveillance-report. Accessed 27 May 2020.

63. McGlothlin AE, Lewis RJ. Minimal clinically important difference: defining what really matters to patients. JAMA. 2014;312(13):1342-3. https://doi.org/1 0.1001/jama.2014.13128.

64. International Severe Acute Respiratory and Emerging Infection Consortium. COVID-19 Clinical Research Resources 2020 [Available from: https://isaric tghn.org/covid-19-clinical-research-resources/. Accessed 30 May 2020.

\section{Publisher's Note}

Springer Nature remains neutral with regard to jurisdictional claims in published maps and institutional affiliations. 